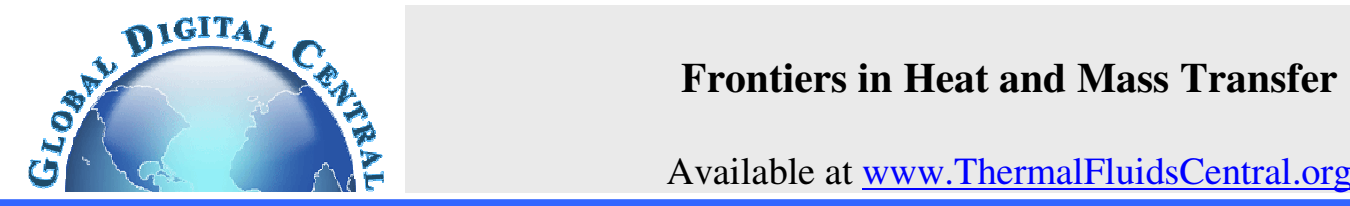

\title{
MHD MAXWELL FLUID FLOW IN PRESENCE OF NANO-PARTICLE THROUGH A VERTICAL POROUS-PLATE WITH HEAT-GENERATION, RADIATION ABSORPTION AND CHEMICAL REACTION
}

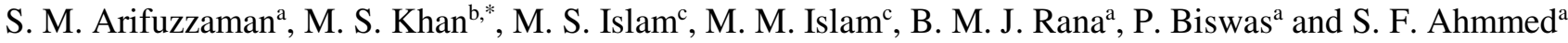 \\ ${ }^{a}$ Mathematics Discipline, Khulna University, Khulna 9208, Bangladesh \\ ${ }^{b}$ Discipline of Chemical Engineering, University of Newcastle, Callaghan, NSW 2308, Australia \\ ${ }^{c}$ Bangabandhu Sheikh Mujibur Rahman Science and Technology University, Gopalganj 8100, Bangladesh
}

\begin{abstract}
Present study concerns with the numerical investigation of MHD transient naturally convective and higher order chemically reactive Maxwell fluid with Nano-particle flow through a vertical porous plate with the effects of heat generation and radiation absorption. A boundary layer approximation is carried out to develop a flow model representing time dependent momentum, energy, and concentration equations. The governing model equations in partial differential equations (PDEs) form are transformed into a set of nonlinear ordinary differential equation (ODEs) by using non-similar technique. Explicit Finite Difference Method (EFDM) is employed by implementing an algorithm in Compaq Visual Fortran 6.6a to solve the obtained set of nonlinear coupled ODEs. For optimizing the system parameter and accuracy of the system, the stability and convergence analysis (SCA) are carried out. It is observed that with initial boundary conditions, $U=V=T=C=0$ and for $\Delta \tau=0.005, \Delta X=0.20$ and $\Delta Y=0.25$, the system converged at Prandtl number, $P_{r} \geq 0.209$ and Lewis number, $\mathrm{L}_{\mathrm{e}} \geq 0.16$. The velocity, temperature and concentration flow are investigated and shown graphically with the effect of system parameters. Furthermore, the effect of system parameters on skin friction coefficient, $C_{f}$, Nusselt number, $N_{u}$, and Sherwood number, $S_{h}$, are also examined and tabularized.
\end{abstract}

Keywords: Maxwell fluid, Nano-particle, MHD, thermal radiation and radiation absorption.

\section{INTRODUCTION}

In the last few decades, the study of magnetohydrodynamic (MHD) heat-mass transfer flow finds vast significant importance in engineering technology industries. A wide range of applications can be found in several fields such as aerodynamic extrusion of plastic sheets, energy storage units, biological transportation, liquid metal fluids oil reservoirs, high-temperature plasmas, geothermal systems, heat insulation and metal and polymer extrusion, thermal energy storage devices, electronic cooling, boilers, nuclear process, micro MHD pumps, ground water systems etc. Investigations of MHD flows are mostly in vertical moving porous plate (Mohamed and Abo-Dahab, 2009), vertical porous plate (Islam et al. 2015; Khan et al. 2014; Murthy et al. 2015; Ramachandra et al. 2011), vertical insulated porous plate (Baoku et al. 2013), infinite inclined porous plate (Das et al. 2015), semi-infinite vertical porous plate (Ravikumar et al. 2014) etc.

In recent days, nanotechnology has received a lot of attention (Bég et al. 2014; Dogonchi and Ganji, 2016; Ferdows et al. 2013; Ibáñez et al. 2016; Kandasamy et al. 2016; Mosayebidorcheh et al. 2016; Pandey et al. 2016; Shahmohamadi and Rashidi, 2016; Sheikholeslami et al., 2016; Biswas et al, 2017; Arifuzzaman et al. 2017; Biswas et al, 2018) where the further development of higher performance is still going due to effective applications in the field of cooling (transformer cooling, electronics device cooling, silicon mirror cooling, vehicles cooling, controlling fusion), biomedical (magnetic cell separation, drug delivery, cancer therapeutics, cryopreservation, nanocryosurgery) etc. The term "nanofluid" can be refers to a class of fluids by suspending nanometre sized (1-100 nm diameters) particles in common base fluids of highly enhanced thermal properties. This type of fluids has highly industrial importance because of its unique chemical and physical properties. It has a higher thermal conductivity which controlled significant enhancement due to the rate of heat transfer.

Maxwell fluid model is one of the subclass of rate type fluids which has gained wide attractions among the researchers in last 10 years. The fluid of both viscosity and elasticity properties is so called Maxwell fluid. The main advantage of using this kind of fluids is it can predict the stress relaxation whilst other differential-type fluids cannot predict such effects. Maxwell fluid widely used in the field of viscoelastic fluid in where the relaxation time (dimensionless) is insignificant however, it's beneficial for significant relaxation time in concentrated polymeric fluids of low molecular weight (Fetecau and Zierep, 2003). Hayat and Sajid (2007) studied hydrodynamics of MHD flow of an upper-convected Maxwell fluid by a Homotopy analysis method. The upper-convected Maxwell fluid flow past a porous shrinking sheet was investigated by Hayat et al. (2008). Hayat and coauthors also considers Maxwell fluid flow in a rotating frame (Hayat et al. 2008), over a stretching surface (Hayat et al. 2009), effect of thermal radiation, thermophoresis and Joule heating on Maxwell fluid flow (Hayat and Qasim, 2010), channel flow of Maxwell fluid (Hayat et al. 2011) in their investigation. Chen et al. (2015) analysed hydrodynamics of Maxwell fluid flow over an unsteady stretching surface. The study of MHD Maxwell fluid flow over a stretching sheet with nanoparticles and presence of chemical reaction was reported by Afify and Elgazery

*Corresponding author. Email: Md.S.Khan@newcastle.edu.au 
(2016). Khan et al. (2016) investigated MHD heat and mass transfer axisymmetric chemically reactive Maxwell fluid flow of driven by exothermal and isothermal stretching disks. In the presence of nano particles, Ramesh et al. (2016) studied Maxwell fluid stagnation point flow of near a permeable surface. Recently, the radiation and viscous dissipation effects on Maxwell fluid flow in a combined MHD heat transfer thermal extrusion system was studied by Hsiao (2017). The rising needs of chemical reactions led the higher importance of investigating chemical reaction influence on MHD heat and mass transfer flow in chemical engineering and hydrometallurgical industries. This study finds vast applications in the field of evaporation, flow in a desert cooler, energy transfer in a cooling tower, drying etc. The effects of chemical reaction on heat and mass transfer MHD boundary layer laminar type flow over a wedge considering suction/injection was analysed by Devi et al. (2002). Kandasamy et al. (2005) investigated the effect of presence of thermal stratification and heat source on chemical reactive fluid flow through a stretching surface. Elgazery (2009) reported the influence of chemical reaction on MHD temperature dependent viscosity and thermal diffusive flow with Hall and ion-slip currents. Zhang and Zheng (2012) examined MHD thermosolutal Marangoni convection flow with incorporation of firstorder chemical reaction. For controlling the hydrodynamics fluid flow behaviour, this study still receiving a lot of consideration in recent years (Eid, 2016; Mabood et al. 2015; Muthuraj et al. 2016; Nayak et al. 2016; Rahman et al. 2014; Reddy, 2016; Reddy et al. 2017; Srinivas et al. 2016).

Now a day, the study of radiation absorption effects on boundary layer fluid flow is industrially important because of manufacturing applications such as catalytic reactors, reactor safety, oil reservoirs, heat insulation, geothermal systems etc. The effect of radiation absorption on transient MHD free convection flow was investigated by Ibrahim et al. (2008). Rubio Hernández (2013) studied the influence of radiation absorption on MHD time dependent fluid flow through a porous medium. MHD micropolar fluid flow in a rotating system with the presence of radiation absorption was investigated by Satya Narayana (2013). Reddy et al. (2015) reported a study concerning with MHD free convective flow with the effect of radiation absorption. More recent studies can have found in the literature (Durga et al. 2016; Satya, 2015; Sreedevi et al. 2016; Umamaheswar et al. 2016).

The effect of heat generation on boundary layer fluid flow is very significant due to engineering applications such as fire and combustion, metal waste, radioactive materials, reactor safety analysis, spent nuclear fuel, etc. Several investigators (Benos et al. 2014; Ganga et al. 2015; Hady et al. 2006; Nandy and Mahapatra, 2013) find out heat generation as an important arena of fluid dynamics research. The heat generation/absorption effects on $\mathrm{MHD}$ convective $\mathrm{Al}_{2} \mathrm{O}_{3}$-water and $\mathrm{TiO}_{2}$-water nanofluids flow past a stretching sheet in porous media was analysed by Reddy et al. (2016) reported. Srinivasa and Eswara (2016) studied heat generation effect of electrically conducting fluid flow towards an isothermal truncated cone. Hayat et al. (2017) analytically investigated the effect of existence of heat generation/absorption on MHD three-dimensional Oldroyd-B nanofluid flow.

To the best of the author's knowledge, the study of natural convective and chemically reactive Maxwell fluid flow with nanoparticle through a vertical porous plate in presence of thermal radiation, heat generation and radiation absorption has remained unexplored. Therefore, this phenomenon is addressed in this study. The specific objectives of this numerical investigation are listed below:

a) To investigate unsteady chemically reactive Maxwell fluid flow with nano particle through a vertical porous plate with the influence of thermal radiation, mass diffusion with heat source, mass transfer and radiation absorption.

b) Mathematical solution of the flow governing model which includes transient momentum, energy and diffusion balance equations numerically using well-known explicit finite difference method (EFDM). c) Optimizing the numerical flow parameters and predicting high accuracy of EFDM solutions by analyzing stability and convergence analysis (SCA).

d) To study velocity, temperature, and concentration distribution across the boundary layer. Investigation on skin friction coefficient, Nusselt number and Sherwood number with different physical parameters.

e) Evaluation of the thermal and momentum boundary layer thickness with isotherms and streamlines analysis.

\section{MATHEMATICAL MODEL OF FLOW}

Unsteady heat and mass transfer flow of Maxwell fluid along a semiinfinite vertical porous plate $(\mathrm{y}=0)$ is considered in the presence of a uniform thermal radiation and magnetic field. The fluid is flowing in the $\mathrm{x}$ - direction which is taken along the plate in the upward $x$-direction and $y$-axis is normal to it. When, the plate velocity $U(t)$ is given as $\mathrm{u}=\mathrm{U}_{0}$.In initial step, it is considered that the plate as well as the fluid particle is at rest at the same temperature $\mathrm{T}\left(=\mathrm{T}_{\infty}\right)$ and the same concentration level $\mathrm{C}\left(=\mathrm{C}_{\infty}\right)$ at all points. Where, $\mathrm{C}_{\infty}$ and $\mathrm{T}_{\infty}$ are fluid concentration and temperature species of uniform flow respectively.
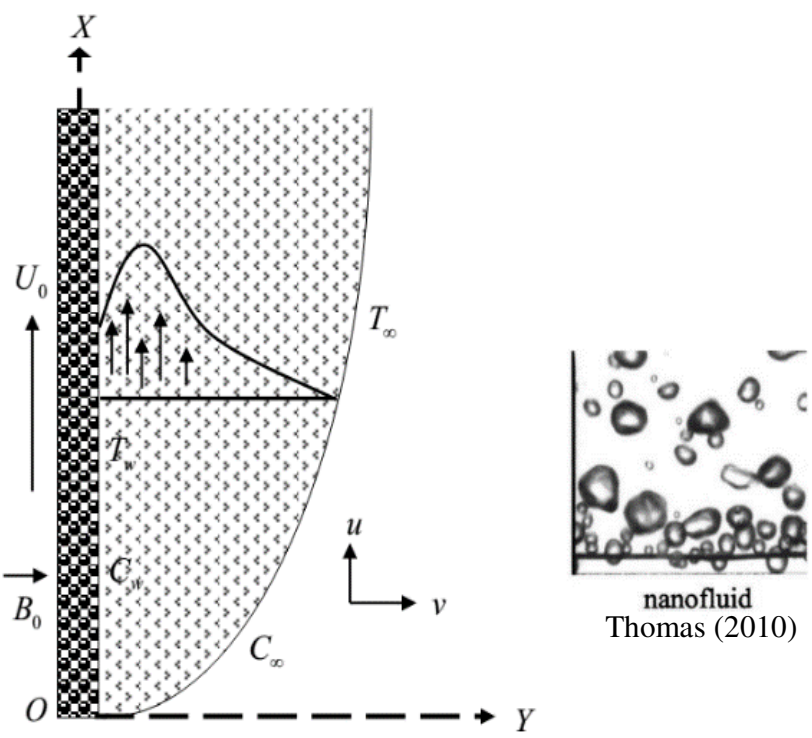

Fig. 1. Physical configuration and coordinate system.

It is also assumed that a magnetic field $B_{y}=B_{o}$ of uniform strength is applied normal to the flow region. The physical configuration and coordinate system of the problem is presented in the following Fig. 1.

Under the assumptions made above, the governing equations for unsteady MHD transient Maxwell fluid flow in presence of Nanoparticle through a vertical porous plate with the effect of chemical reaction, heat generation and radiation absorption into account, reduce to:

Continuity Equation,

$\frac{\partial \mathrm{u}}{\partial \mathrm{x}}+\frac{\partial \mathrm{v}}{\partial \mathrm{y}}=0$

Momentum Equation,

$\frac{\partial u}{\partial t}+u \frac{\partial u}{\partial x}+v \frac{\partial u}{\partial y}=v \frac{\partial^{2} u}{\partial x^{2}}-\lambda_{1}\left[u^{2} \frac{\partial^{2} u}{\partial x^{2}}+v^{2} \frac{\partial^{2} u}{\partial y^{2}}+2 u v \frac{\partial^{2} u}{\partial x \partial y}\right]$
$-\frac{\sigma B_{0}^{2}}{\rho} u+g \beta\left(T-T_{\infty}\right)+g \beta^{*}\left(C-C_{\infty}\right)-\frac{v}{k} u$ 


\section{Energy Equation,}

$$
\begin{aligned}
& \frac{\partial \mathrm{T}}{\partial \mathrm{t}}+\mathrm{u} \frac{\partial \mathrm{T}}{\partial \mathrm{x}}+\mathrm{v} \frac{\partial \mathrm{T}}{\partial \mathrm{y}}=\frac{\kappa \partial^{2} \mathrm{~T}}{\rho \mathrm{c}_{\mathrm{p}} \partial \mathrm{y}^{2}}+\frac{\mathrm{v}}{\mathrm{c}_{\mathrm{p}}}\left(\frac{\partial \mathrm{u}}{\partial \mathrm{y}}\right)^{2}+\frac{\mathrm{Q}_{0}}{\rho \mathrm{c}_{\mathrm{p}}}\left(\mathrm{T}-\mathrm{T}_{\infty}\right)+\frac{\mathrm{Q}_{1}^{*}}{\rho \mathrm{c}_{\mathrm{p}}}\left(\mathrm{C}-\mathrm{C}_{\infty}\right) \\
& -\frac{1}{\rho \mathrm{c}_{\mathrm{p}}} \frac{\partial \mathrm{q}_{\mathrm{r}}}{\partial \mathrm{y}}+\frac{\mathrm{D}_{\mathrm{m}} \kappa_{\mathrm{T}}}{\mathrm{c}_{\mathrm{s}} \mathrm{c}_{\mathrm{p}}} \frac{\partial^{2} \mathrm{C}}{\partial \mathrm{y}^{2}}+\frac{\sigma \mathrm{B}_{0}^{2} \mathrm{u}^{2}}{\rho \mathrm{c}_{\mathrm{p}}}+\Gamma\left\{\mathrm{D}_{\mathrm{B}}\left(\frac{\partial \mathrm{T}}{\partial \mathrm{y}} \frac{\partial \mathrm{C}}{\partial \mathrm{y}}\right)+\frac{\mathrm{D}_{\mathrm{T}}}{\mathrm{T}_{\infty}}\left(\frac{\partial \mathrm{T}}{\partial \mathrm{y}}\right)^{2}\right\}
\end{aligned}
$$

\section{Concentration Equation,}

$$
\frac{\partial C}{\partial t}+u \frac{\partial C}{\partial x}+v \frac{\partial C}{\partial y}=D_{B}\left(\frac{\partial^{2} C}{\partial y^{2}}\right)+\frac{D_{T}}{T_{\infty}} \frac{\partial^{2} T}{\partial y^{2}}-K_{c}\left(C-C_{\infty}\right)^{P}
$$

\section{With boundary condition,}

$$
\mathrm{u}=\mathrm{U}_{0}, \mathrm{~T}=\mathrm{T}_{\mathrm{w}}, \mathrm{C}=\mathrm{C}_{\mathrm{w}} \text { at } \mathrm{y}=0
$$$$
\mathrm{u}=0, \mathrm{~T}=\mathrm{T}_{\infty}, \mathrm{C}=\mathrm{C}_{\infty} \text { at } \mathrm{y} \rightarrow \infty
$$

Where, $\mathrm{u}$ and $\mathrm{v}$ are the velocity component, $B_{0}$ is the magnetic field component, $\beta$ is thermal expansion coefficient, $\beta^{*}$ is concentration expansion coefficient, $T_{w}$ denotes the wall temperature, $C_{w}$ is the species concentration at the wall, $v$ is the kinematic viscosity, $\rho$ is density, $\kappa$ is thermal conductivity, $c_{p}$ is specific heat at constant pressure, $Q_{0}$ denotes the heat source, $Q_{1}^{*}$ denotes the radiation absorption, $q_{r}$ unidirectional radiative heat flux, $K_{c}$ for chemical reaction, $D_{B}$ is Brownian diffusion coefficient, $D_{T}$ thermophoresis diffusion coefficient and $\lambda_{1}$ is the relaxation time of the upperconvected Maxwell (UCM) fluid. The radiative heat flux term by using the Rosseland approximation is given by $q_{r}=-\frac{4 \sigma_{s}}{3 k_{e}} \frac{\partial T^{4}}{\partial y}$.

Where, $\sigma_{s}$ is the Stefan-Boltzmann constant and $k_{e}$ is the mean absorption coefficient, respectively. If temperature differences within the flow are sufficiently small, then the $q_{r}$ can be linearized by expanding $T^{4}$ into the Taylor series about $T_{\infty}$, which after neglecting higher order terms takes the form by $T^{4} \cong 4 T_{\infty}{ }^{3} T-3 T_{\infty}^{4}$. Then the equation (3) becomes,

$$
\begin{aligned}
& \frac{\partial T}{\partial t}+u \frac{\partial T}{\partial x}+v \frac{\partial T}{\partial y}=\frac{\kappa \partial^{2} T}{\rho c_{p} \partial y^{2}}+\frac{v}{c_{p}}\left(\frac{\partial u}{\partial y}\right)^{2}+\frac{Q_{0}}{\rho c_{p}}\left(T-T_{\infty}\right)+\frac{Q_{1}^{*}}{\rho c_{p}}\left(C-C_{\infty}\right) \\
& +\frac{16 \sigma_{s} T_{\infty}^{3}}{3 k_{e} \rho c_{p}} \frac{\partial^{2} T}{\partial y^{2}}+\frac{D_{m} \kappa_{T}}{c_{s} c_{p}} \frac{\partial^{2} C}{\partial y^{2}}+\frac{\sigma B_{0}^{2} u^{2}}{\rho c_{p}}+\Gamma\left\{D_{B}\left(\frac{\partial T}{\partial y} \frac{\partial C}{\partial y}\right)+\frac{D_{T}}{T_{\infty}}\left(\frac{\partial T}{\partial y}\right)^{2}\right\}
\end{aligned}
$$

From the governing equations (1) - (5) under the initial conditions and the boundary conditions will be based on the finite difference method it is required to make the equations dimensionless. For the purpose introducing the following dimensionless quantities:

$X=\frac{x U_{0}}{v}, Y=\frac{y U_{0}}{v}, U=\frac{u}{U_{0}}, V=\frac{v}{U_{0}}, \tau=\frac{t U_{0}^{2}}{v}, \theta=\frac{T-T_{\infty}}{T_{w}-T_{\infty}}, \phi=\frac{C-C_{\infty}}{C_{w}-C_{\infty}}$,

So, $x=\frac{X v}{U_{0}}, y=\frac{Y v}{U_{0}}, u=U U_{0}, t=\frac{\tau v}{U_{0}^{2}}, T=T_{\infty}+\theta\left(T_{w}-T_{\infty}\right)$ and

$C=C_{\infty}+\phi\left(C_{w}-C_{\infty}\right)$,

the dimensionless equations are obtained as follows:

Dimensionless Continuity Equation,

$U \frac{\partial U}{\partial X}+V \frac{\partial U}{\partial Y}=0$

\section{Dimensionless Momentum Equation,}

$\frac{\partial U}{\partial \tau}+U \frac{\partial U}{\partial X}+V \frac{\partial U}{\partial Y}=\frac{\partial^{2} U}{\partial Y^{2}}-\mathrm{N}_{v}\left[U^{2} \frac{\partial^{2} U}{\partial X^{2}}+V^{2} \frac{\partial^{2} U}{\partial Y^{2}}+2 U V \frac{\partial^{2} U}{\partial X \partial Y}\right]_{(7)}$
$+G_{r} \theta+G_{c} \phi-M U-\frac{1}{D_{a}} U$

Dimensionless Energy Equation,

$\frac{\partial \theta}{\partial \tau}+\frac{\partial \theta}{\partial Y} U+V \frac{\partial \theta}{\partial Y}=\frac{1}{P_{r}}\left(1+\frac{16 R}{3}\right) \frac{\partial^{2} \theta}{\partial Y^{2}}+Q \theta+Q_{1} \phi+E_{c}\left(\frac{\partial U}{\partial Y}\right)^{2}+D_{u} \frac{\partial^{2} \phi}{\partial Y^{2}}$

$+E_{c} M U^{2}+N_{b}\left(\frac{\partial \theta}{\partial Y} \frac{\partial C}{\partial Y}\right)+N_{t}\left(\frac{\partial \theta}{\partial Y}\right)^{2}$

Dimensionless Concentration Equation,

$$
\begin{aligned}
& \frac{\partial \phi}{\partial \tau}+U \frac{\partial \phi}{\partial X}+V \frac{\partial \phi}{\partial Y}=\frac{1}{L_{e}}\left[\frac{\partial^{2} \phi}{\partial Y^{2}}+\left(\frac{N_{t}}{N_{b}}\right) \frac{\partial^{2} \theta}{\partial Y^{2}}\right]-K_{r} \phi^{P} \\
& U=1, \theta=1, \varphi=1 \quad \text { at } y=0 \\
& U=0, \theta=0, \varphi=0 \text { at } y \rightarrow \infty
\end{aligned}
$$

Where the obtained physical parameters are given below:

magnetic parameter, $M=\frac{\sigma^{\prime} B_{0}^{2} v}{\rho U_{0}^{2}}$, Darcy number, $D_{a}=\frac{K^{\prime} U_{0}^{2}}{v^{2}}$, Prandtl number, $P_{r}=\frac{\rho c_{p} v}{\kappa}$, radiation parameter, $R=\frac{\sigma T_{\infty}^{3}}{k_{e} \kappa}$, heat source parameter, $Q=\frac{Q_{0} v}{U_{0}^{2} \rho c_{p}}$, radiation absorption parameter, $Q_{1}=\frac{Q_{1}^{*} v}{U_{0}^{2} \rho c_{p}}$,

Eckert number, $\quad E_{c}=\frac{U_{0}^{2}}{c_{p}\left(T_{w}-T_{\infty}\right)}, \quad$ Dufour number, $D_{u}=\frac{D_{m} \kappa_{T}}{c_{s} c_{p} v}\left(\frac{C_{w}-C_{\infty}}{T_{w}-T_{\infty}}\right), \quad$ Lewis $\quad$ number, $\quad L_{e}=\frac{v}{D_{m}}$, Maxwell parameter, $N_{v}=\frac{\lambda_{1} U_{0}^{2}}{v}$, Brownian parameter, $N_{b}=\frac{\Gamma D_{B}\left(C_{w}-C_{\infty}\right)}{v}$, thermophoresis parameter, $N_{t}=\frac{\Gamma D_{T}}{T_{\infty} v}\left(T_{w}-T_{\infty}\right)$, Order of chemical reaction and $=\mathrm{P}$, chemical reaction, $K_{r}=\frac{v K_{c}\left(C_{w}-C_{\infty}\right)^{p-1}}{U_{0}^{2}}$.

Stream function $\psi$ satisfies the continuity Eq. (6) and is associated with the velocity components in the usual way as,

$U=\frac{\partial \psi}{\partial Y}, V=-\frac{\partial \psi}{\partial X}$

The parameters of technological interest for the present problem are the local skin-friction, the local Nusselt number and the local Sherwood number, which are elucidated as;

$$
\begin{aligned}
& C_{f}=-\frac{1}{2 \sqrt{2}} G_{r}^{-3 / 4}\left(\frac{\partial U}{\partial Y}\right)_{Y=0} \\
& N_{u}=\frac{1}{\sqrt{2}} G_{r}^{-3 / 4}\left(\frac{\partial \theta}{\partial Y}\right)_{Y=0} \\
& S_{h}=\frac{1}{2 \sqrt{2}} G_{r}^{-3 / 4}\left(\frac{\partial \phi}{\partial Y}\right)_{Y=0}
\end{aligned}
$$

\section{NUMERICAL TECHNIQUE}

To solve the governing coupled non-dimensional partial differential equations with the associated initial and boundary conditions. The method of explicit finite difference has been used to solve (6) - (9) subject to the initial and boundary conditions. For this reason, the area 
within the boundary layer is divided by some perpendicular lines of $Y$ axis, where the normal of the medium is $Y$ - axis as shown in Fig. 2. It is assumed that the maximum length of boundary layer $Y_{\max }=20$ as corresponds to $Y \rightarrow \infty$. i.e. $Y$ vary from 0 to 20 and the number of grid spacing in $Y$ directions are $m(=100)$ and $n(=200)$, with the smaller time step $\Delta \tau=0.005$. Using the explicit finite difference approximation, we have,

\section{Continuity Equation,}

$\frac{U_{i, j}-U_{i-1, j}}{\Delta X}+\frac{V_{i, j}-V_{i, j-1}}{\Delta Y}=0$

Momentum Equation

$$
\begin{aligned}
& \frac{U_{i, j}^{\prime}-U_{i, j}}{\Delta \tau}+U_{i, j} \frac{U_{i, j}-U_{i-1, j}}{\Delta X}+V_{i, j} \frac{U_{i, j+1}-U_{i, j}}{\Delta Y}=\frac{U_{i, j+1}-2 U_{i, j}+U_{i, j-1}}{(\Delta Y)^{2}} \\
& -N_{v}\left[\left(U_{i, j}\right)^{2} \frac{U_{i, j+1}-2 U_{i, j}+U_{i, j-1}}{(\Delta X)^{2}}+\left(V_{i, j}\right)^{2} \frac{U_{i, j+1}-2 U_{i, j}+U_{i, j-1}}{(\Delta Y)^{2}}\right. \\
& \left.+\left(2 U_{i, j} V_{i, j} \frac{U_{i+1, j+1}-U_{i+1, j-1}-U_{i-1, j+1}+U_{i-1, j-1}}{4 \Delta X \Delta Y}\right)\right] \\
& +G_{r} \theta_{i, j}+G_{c} \phi_{i, j}-\left(M+\frac{1}{D_{a}}\right) U_{i, j}
\end{aligned}
$$

\section{Energy Equation,}

$$
\begin{aligned}
& \frac{\theta_{i, j}^{\prime}-\theta_{i, j}}{\Delta \tau}+U_{i, j} \frac{\theta_{i, j}-\theta_{i-1, j}}{\Delta X}+V_{i, j} \frac{\theta_{i, j+1}-\theta_{i, j}}{\Delta Y}=\frac{1}{P_{r}}\left(1+\frac{16}{3} R\right) \frac{\theta_{i, j+1}-2 \theta_{i, j}+\theta_{i, j-1}}{(\Delta Y)^{2}} \\
& +D_{u} \frac{\phi_{i, j+1}-2 \phi_{i, j}+\phi_{i, j-1}}{(\Delta Y)^{2}}+Q \theta_{i, j}+Q_{1} \phi_{i, j}+E_{c}\left(\frac{U_{i, j+1}-U_{i, j}}{\Delta Y}\right)^{2}+E_{c} M\left(U_{i, j}\right)^{2} \\
& +N_{b}\left(\frac{\theta_{i, j+1}-\theta_{i, j}}{\Delta Y} \cdot \frac{\varphi_{i, j+1}-\varphi_{i, j}}{\Delta Y}\right)+N_{t}\left(\frac{\theta_{i, j+1}-\theta_{i, j}}{\Delta Y}\right)^{2}
\end{aligned}
$$

\section{Concentration Equation,}

$$
\begin{aligned}
& \frac{\phi_{i, j}^{\prime}-\phi_{i, j}}{\Delta \tau}+U_{i, j} \frac{\phi_{i, j}-\phi_{i-1, j}}{\Delta X}+V_{i, j} \frac{\phi_{i, j+1}-\phi_{i, j}}{\Delta Y}=\frac{1}{L_{e}}\left[\frac{\phi_{i, j+1}-2 \phi_{i, j}+\phi_{i, j-1}}{(\Delta Y)^{2}}\right. \\
& \left.+\left(\frac{N_{t}}{N_{b}}\right) \frac{\theta_{i, j+1}-2 \theta_{i, j}+\theta_{i, j-1}}{(\Delta Y)^{2}}\right]-K_{r}\left(\phi_{i, j}\right)^{P}
\end{aligned}
$$

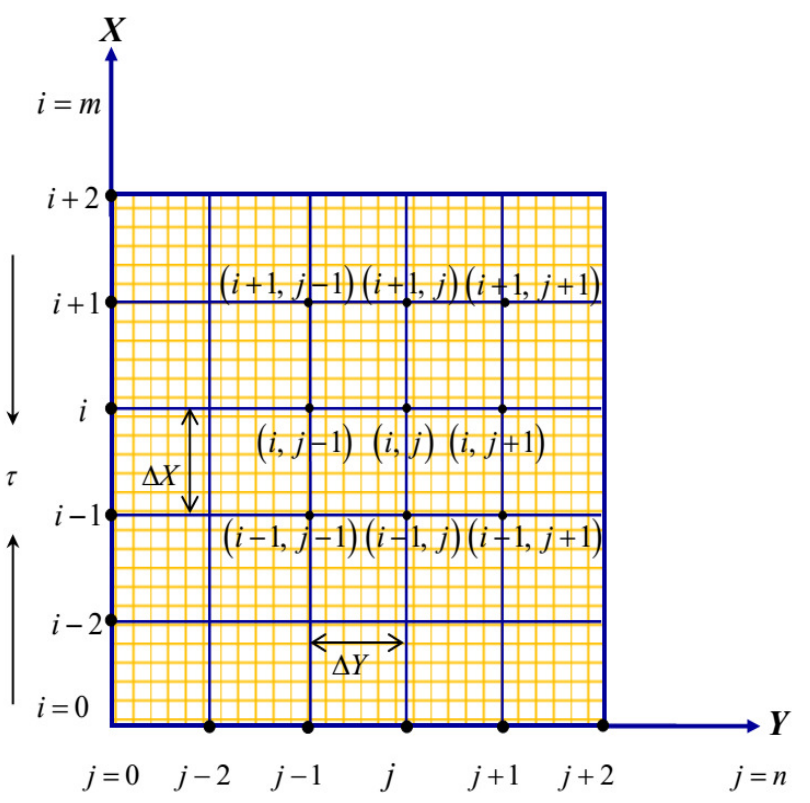

Fig. 2 The finite difference space grid.
The initial and boundary condition with finite difference scheme as

$U_{i, 0}^{n}=1, \theta_{i, 0}^{n}=1, \phi_{i, 0}^{n}=1$

$U_{i, L}^{n}=0, \theta_{i, L}^{n}=0, \phi_{i, L}^{n}=0$ Where, $L \rightarrow \infty$

Where, the subscripts $i$ and $j$ designate the grid points with $X$ and $Y$ coordinates respectively and a value of time, $\tau=n \Delta \tau$, where $n=1,2,3,4 \ldots \ldots$.

\section{STABILITY AND CONVERGENCE ANALYSIS OF THE PROBLEM}

For the solution an explicit finite difference approach is being used therefore the analysis is remained incomplete unless the stability and convergence of the finite difference scheme are discussed. For the constant mesh size, the stability criteria of the scheme may be established as follows. The general terms of the Fourier expansion for $U, \theta$ and $\varphi$ at a time arbitrarily called $t=0$ are all $e^{i \alpha X} e^{i \beta Y}$ apart from a constant, where $i=\sqrt{-1}$. A time $t=\tau$, these terms becomes

$$
\left.\begin{array}{l}
U: \psi(\tau) e^{i \alpha X} e^{i \beta Y} \\
\theta: \theta(\tau) e^{i \alpha X} e^{i \beta Y} \\
\varphi: \varphi(\tau) e^{i \alpha X} e^{i \beta Y}
\end{array}\right\}
$$

after a time step these terms convert to

$$
\left.\begin{array}{l}
U: \psi^{\prime}(\tau) e^{i \alpha X} e^{i \beta Y} \\
\theta: \theta^{\prime}(\tau) e^{i \alpha X} e^{i \beta Y} \\
\varphi: \varphi^{\prime}(\tau) e^{i \alpha X} e^{i \beta Y}
\end{array}\right\}
$$

Substituting (17) and (18) to the main (13) -(16) equation we get,

$$
\begin{aligned}
& \psi^{\prime}=\psi(\tau)+\Delta \tau\left[G_{r} \theta+G_{c} \varphi-\left(M+\frac{1}{D_{a}}\right) \psi+\frac{2(\cos \beta \Delta Y-1)}{(\Delta Y)^{2}} \psi\right. \\
& -\frac{U\left(1-e^{i \alpha \Delta X}\right)}{\Delta X} \psi-\frac{V\left(e^{i \beta \Delta Y}-1\right)}{\Delta Y} \psi-N_{v}\left[\frac{2 U^{2} \psi(1-\cos \alpha \Delta X)}{(\Delta X)^{2}}\right. \\
& +\frac{2 V^{2} \psi(\cos \beta \Delta Y-1)}{(\Delta Y)^{2}} \\
& +2 \psi U V \frac{e^{i \alpha(X+\Delta X)} e^{i \beta(Y+\Delta Y)}-e^{i \alpha(X+\Delta X)} e^{i \beta(Y-\Delta Y)}}{4 \Delta X \Delta Y} \\
& \left.\left.-2 \psi U V \frac{\left(e^{i \alpha(X-\Delta X)} e^{i \beta(Y+\Delta Y)}-e^{i \alpha(X-\Delta X)} e^{i \beta(Y-\Delta Y)}\right)}{4 \Delta X \Delta Y}\right]\right] \\
& \Rightarrow \psi \psi^{\prime}=\left[1+\Delta \tau \frac{2(\cos \beta \Delta Y-1)}{(\Delta Y)^{2}}-\Delta \tau\left(M+\frac{1}{D_{a}}\right)-\frac{U \Delta \tau\left(1-e^{i \alpha \Delta X}\right)}{\Delta X}\right. \\
& -\frac{V \Delta \tau\left(e^{i \beta \Delta Y}-1\right)}{\Delta Y}+\Delta \tau G_{r} \theta+\Delta \tau G_{c} \varphi \\
& -N_{v}\left[\frac{2 U^{2} \Delta \tau(1-\cos \alpha \Delta X)}{(\Delta X)^{2}}+\frac{2 V^{2} \Delta \tau(\cos \beta \Delta Y-1)}{(\Delta Y)^{2}}\right. \\
& \left.\left.+2 U V \Delta \tau \frac{e^{i \alpha(X+\Delta X)} e^{i \beta(Y+\Delta Y)}-e^{i \alpha(X+\Delta X)} e^{i \beta(Y-\Delta Y)}}{4 \Delta X \Delta Y}\right]\right] \psi \\
& -2 U V \Delta \tau \frac{\left(e^{i \alpha(X-\Delta X)} e^{i \beta(Y+\Delta Y)}-e^{i \alpha(X-\Delta X)} e^{i \beta(Y-\Delta Y)}\right)}{4 \Delta X \Delta Y}
\end{aligned}
$$

$\psi^{\prime}=A_{1} \psi+A_{2} \theta+A_{3} \varphi$ 
where

$$
\begin{aligned}
& A_{1}=1+\Delta \tau \frac{2(\cos \beta \Delta Y-1)}{(\Delta Y)^{2}}-\Delta \tau\left(M+\frac{1}{D_{a}}\right)-\frac{U \Delta \tau\left(1-e^{i \alpha \Delta X}\right)}{\Delta X} \\
& -\frac{V \Delta \tau\left(e^{i \beta \Delta Y}-1\right)}{\Delta Y} \\
& -N_{v}\left[\frac{2 U^{2} \Delta \tau(1-\cos \alpha \Delta X)}{(\Delta X)^{2}}+\frac{2 V^{2} \Delta \tau(\cos \beta \Delta Y-1)}{(\Delta Y)^{2}}\right. \\
& +2 U V \Delta \tau \frac{e^{i \alpha(X+\Delta X)} e^{i \beta(Y+\Delta Y)}-e^{i \alpha(X+\Delta X)} e^{i \beta(Y-\Delta Y)}}{4 \Delta X \Delta Y} \\
& \left.-2 U V \Delta \tau \frac{\left(e^{i \alpha(X-\Delta X)} e^{i \beta(Y+\Delta Y)}-e^{i \alpha(X-\Delta X)} e^{i \beta(Y-\Delta Y)}\right)}{4 \Delta X \Delta Y}\right] \\
& A_{2}=\Delta \tau G_{r} \text { and } A_{3}=\Delta \tau G_{c}
\end{aligned}
$$

For temperature equation,

$$
\begin{aligned}
& \theta^{\prime}=\theta+\Delta \tau\left[\frac{1}{P_{r}}\left(1+\frac{16 R}{3}\right) \frac{2 \theta(\cos \beta \Delta Y-1)}{(\Delta Y)^{2}}+D_{u} \frac{2 \varphi(\cos \beta \Delta Y-1)}{(\Delta Y)^{2}}+Q_{1} \varphi\right. \\
& +E_{c} \frac{\left(e^{i \beta \Delta Y}-1\right)^{2}}{\Delta Y^{2}} \psi+Q \theta-\frac{U\left(1-e^{i \alpha \Delta X}\right)}{\Delta X} \psi-\frac{V\left(e^{i \beta \Delta Y}-1\right)}{\Delta Y} \psi \\
& \left.+E_{c} M \psi^{2}+N_{b} \theta C\left\{\frac{e^{i \beta \Delta Y}-1}{\Delta Y}\right\}^{2}+N_{t} T \theta\left\{\frac{e^{i \beta \Delta Y}-1}{\Delta Y}\right\}^{2}\right] \\
& \theta^{\prime}=\theta\left[1+\frac{\Delta \tau}{P_{r}}\left(1+\frac{16 R}{3}\right) \frac{2(\cos \beta \Delta Y-1)}{(\Delta Y)^{2}}+Q \Delta \tau-\frac{U \Delta \tau\left(1-e^{i \alpha \Delta X}\right)}{\Delta X}\right. \\
& \left.-\frac{V \Delta \tau\left(e^{i \beta \Delta Y}-1\right)}{\Delta Y}+N_{t} T \Delta \tau\left\{\frac{e^{i \beta \Delta Y}-1}{\Delta Y}\right\}^{2}+N_{b} C \Delta \tau\left\{\frac{e^{i \beta \Delta Y}-1}{\Delta Y}\right\}^{2}\right] \\
& +\left[Q_{1} \Delta \tau+D_{u} \frac{2 \Delta \tau(\cos \beta \Delta Y-1)}{(\Delta Y)^{2}}\right] \varphi+E_{c} \Delta \tau \frac{U\left(e^{i \beta \Delta Y}-1\right)^{2}}{(\Delta Y)^{2}} \psi+E_{c} \Delta \tau M \psi^{2}
\end{aligned}
$$

For obtaining the stability condition, it should be find out Eigen values of the amplification matrix $\mathrm{T}$, but this study is very difficult since all the elements of $\mathrm{T}$ are different. Hence, the problem requires that the Eckert number $E_{c}$ is assumed to be very small, that is, tends to zero. Then we get,

$$
\begin{aligned}
& \theta^{\prime}=\theta\left[1+\frac{\Delta \tau}{P_{r}}\left(1+\frac{16 R}{3}\right) \frac{2(\cos \beta \Delta Y-1)}{(\Delta Y)^{2}}+Q \Delta \tau-\frac{U \Delta \tau\left(1-e^{i \alpha \Delta X}\right)}{\Delta X}\right. \\
& \left.-\frac{V \Delta \tau\left(e^{i \beta \Delta Y}-1\right)}{\Delta Y}+N_{t} T \Delta \tau\left\{\frac{e^{i \beta \Delta Y}-1}{\Delta Y}\right\}^{2}++N_{b} C \Delta \tau\left\{\frac{e^{i \beta \Delta Y}-1}{\Delta Y}\right\}^{2}\right] \\
& +\left[Q_{1} \Delta \tau+D_{u} \frac{2 \Delta \tau(\cos \beta \Delta Y-1)}{(\Delta Y)^{2}}\right] \varphi \\
& \theta^{\prime}=A_{4} \theta+A_{5} \varphi
\end{aligned}
$$

Where,

$$
\begin{aligned}
& A_{4}=1+\frac{\Delta \tau}{P_{r}}\left(1+\frac{16 R}{3}\right) \frac{2(\cos \beta \Delta Y-1)}{(\Delta Y)^{2}}+Q \Delta \tau-\frac{U \Delta \tau\left(1-e^{i \alpha \Delta X}\right)}{\Delta X} \\
& -\frac{V \Delta \tau\left(e^{i \beta \Delta Y}-1\right)}{\Delta Y}+N_{t} T \Delta \tau\left\{\frac{e^{i \beta \Delta Y}-1}{\Delta Y}\right\}^{2}++N_{b} C \Delta \tau\left\{\frac{e^{i \beta \Delta Y}-1}{\Delta Y}\right\}^{2}
\end{aligned}
$$

and $A_{5}=Q_{1} \Delta \tau+D_{u} \frac{2 \Delta \tau(\cos \beta \Delta Y-1)}{(\Delta Y)^{2}}$.

For the concentration equation,

$$
\begin{gathered}
\varphi^{\prime}=\varphi+\Delta \tau\left[\frac{1}{L_{e}}\left[\frac{2 \varphi(\cos \beta \Delta Y-1)}{(\Delta Y)^{2}}+\frac{N_{t}}{N_{b}} \frac{2(\cos \beta \Delta Y-1)}{(\Delta Y)^{2}} \theta\right]\right. \\
\left.-K_{r} \varphi^{P}-\frac{U\left(1-e^{i \alpha \Delta X}\right)}{\Delta X} \varphi-\frac{V\left(e^{i \beta \Delta Y}-1\right)}{\Delta Y} \varphi\right]
\end{gathered}
$$

$$
\begin{aligned}
\varphi^{\prime}= & \varphi\left[1+\frac{2 \Delta \tau}{S_{c}} \frac{(\cos \beta \Delta Y-1)}{(\Delta Y)^{2}}-\Delta \tau K_{r}-\frac{U \Delta \tau\left(1-e^{i \alpha \Delta X}\right)}{\Delta X}-\frac{V \Delta \tau\left(e^{i \beta \Delta Y}-1\right)}{\Delta Y}\right] \\
& +\frac{N_{t}}{N_{b}} \frac{2(\cos \beta \Delta Y-1)}{(\Delta Y)^{2}} \theta
\end{aligned}
$$$$
\varphi^{\prime}=A_{6} \varphi+A_{7} \theta
$$

Where,

$$
\begin{aligned}
& A_{6}=1+\frac{2 \Delta \tau}{L_{e}} \frac{(\cos \beta \Delta Y-1)}{(\Delta Y)^{2}}-\Delta \tau K_{r}-\frac{U \Delta \tau\left(1-e^{i \alpha \Delta X}\right)}{\Delta X}-\frac{V \Delta \tau\left(e^{i \beta \Delta Y}-1\right)}{\Delta Y} \text { and } \\
& A_{7}=\left(\frac{N_{t}}{N_{b}}\right) \frac{2(\cos \beta \Delta Y-1)}{(\Delta Y)^{2}} .
\end{aligned}
$$

Equation (19) -(21) can be expressed in the Matrix form,

$$
\left[\begin{array}{l}
\psi^{\prime} \\
\theta^{\prime} \\
\varphi^{\prime}
\end{array}\right]=\left[\begin{array}{ccc}
A_{1} & A_{2} & A_{3} \\
0 & A_{4} & A_{5} \\
0 & A_{7} & A_{6}
\end{array}\right]\left[\begin{array}{l}
\psi \\
\theta \\
\varphi
\end{array}\right] \text { i.e. } \eta^{\prime}=T^{\prime} \eta
$$

For obtaining the stability condition, Eigen values of the amplification matrix $T^{\prime}$ should be finding out. It is a forth order square matrix. For this explicit finite difference solution, the dimensionless time difference $\Delta \tau$ is very small i.e. tends to zero. Under this condition, $A_{2} \rightarrow 0, A_{3} \rightarrow 0, A_{5} \rightarrow 0$ and $A_{7} \rightarrow 0$

$$
\therefore T^{\prime}=\left[\begin{array}{ccc}
A_{1} & 0 & 0 \\
0 & A_{4} & 0 \\
0 & 0 & A_{6}
\end{array}\right]
$$

After simplification of the matrix $T^{\prime}$ the Eigen values are follows, The Eigen values of the amplification matrix $T^{*}$ are obtained as $A_{1}=\lambda_{1}, A_{4}=\lambda_{2}$ and $A_{6}=\lambda_{3}$. For stability test, each of the Eigen values must not exceeded unity in modulus. Under this consideration, the stability conditions are as follows

$\left|A_{1}\right| \leq 1,\left|A_{4}\right| \leq 1$ and $\left|A_{6}\right| \leq 1$

Let, $a_{1}=\Delta \tau, b_{1}=U \frac{\Delta \tau}{\Delta X}, c_{1}=|-V| \frac{\Delta \tau}{\Delta X}$ and $d_{1}=2 \frac{\Delta \tau}{(\Delta Y)^{2}}$ then,

The coefficient of $\mathrm{a}, \mathrm{b}$ and $\mathrm{c}$ are all non-negative. So the maximum modulus of $A_{1}, A_{4}$ and $A_{6}$ occurs when $\alpha \Delta Y=n \pi$, where $n$ is integer and hence $A_{1}, A_{4}$ and $A_{6}$ are real. The values of $\left|A_{1}\right|,\left|A_{4}\right|$ and $\left|A_{6}\right|$ are greater when $n$ is odd integer, in which case;

$$
\begin{aligned}
& A_{4}=1-2\left[d_{1} \frac{1}{P_{r}}\left(1+\frac{4}{3} R\right)+\frac{a_{1}}{2} Q+b_{1}+c_{1}+2 N_{b} C d_{1}+2 N_{t} T d_{1}\right] \\
& A_{6}=1-2\left[d_{1} \frac{1}{L_{e}}+b_{1}+c_{1}+\frac{a_{1}}{2} \mathrm{~K}_{c}\right]
\end{aligned}
$$

To satisfied allowable values are $A_{1}=-1 \quad A_{4}=-1$ and $A_{6}=-1$. Hence the stability conditions of the methods are,

$U \frac{\Delta \tau}{\Delta X}+V \frac{\Delta \tau}{\Delta X}+\frac{2}{P_{r}}\left(1+\frac{16}{3} R\right) \frac{\Delta \tau}{(\Delta Y)^{2}}-\frac{\Delta \tau Q}{2}+2 N_{b} \frac{\Delta \tau}{(\Delta Y)^{2}}+2 N_{t} \frac{\Delta \tau}{(\Delta Y)^{2}} \leq 1$

and, $U \frac{\Delta \tau}{\Delta X}+V \frac{\Delta \tau}{\Delta X}+\frac{2}{L_{e}} \frac{\Delta \tau}{(\Delta Y)^{2}}+\frac{\Delta \tau K_{r}}{2} \leq 1$.

With initial boundary conditions $U=V=T=C=0$ and for the values of $\Delta \tau=0.005, \Delta X=0.20$ and $\Delta Y=0.25$ then the problem will be converged at $P_{r} \geq 0.209$ and $L_{e} \geq 0.16$. These converge solutions are shown graphically in Figs. 3-19.

\section{RESULTS AND DISCUSSION}

To investigate the physical phenomena of the problem, a finite difference solution is obtained by the use of an explicit procedure. The 
numerical values of non-dimensional velocity, temperature and concentration within the boundary layer for different values of nondimensional parameter have been computed by a FORTRAN program. For the steady-state solutions, the computations have been carried out up to dimensionless time $\tau=30$. (see Appendix A) Porous flow is always very with low Reynolds number so any inertial effects are practically non-existent. Porosity to gases are different than those for liquids in the same media. In Fig. 3(a) and Fig. 3(b)the velocity and skin friction profiles increase in case of strong Darcy number $\left(D_{a}\right)$ and become zero and for the skin friction profiles it remains unchanged with in short range for same values of Darcy number $\left(D_{a}\right)$. This parameter has a very strong physical significance on the fluid velocity. For the values of $D_{a} \geq 0.50$, the fluid velocity increases rapidly. The numerical value obtained from the EFDM simulation for Darcy number $\left(D_{a}\right)$ varies from 0.50 to 3.0 the curve to curve fluctuations of velocity profiles are $207.328 \%, 76.833 \%$ and $28.597 \%$ increase at point $\mathrm{Y}=$ 3.00. On the other hand, skin friction increases with $63.52 \%, 26.562 \%$ and $11.737 \%$ at $\tau=10$.
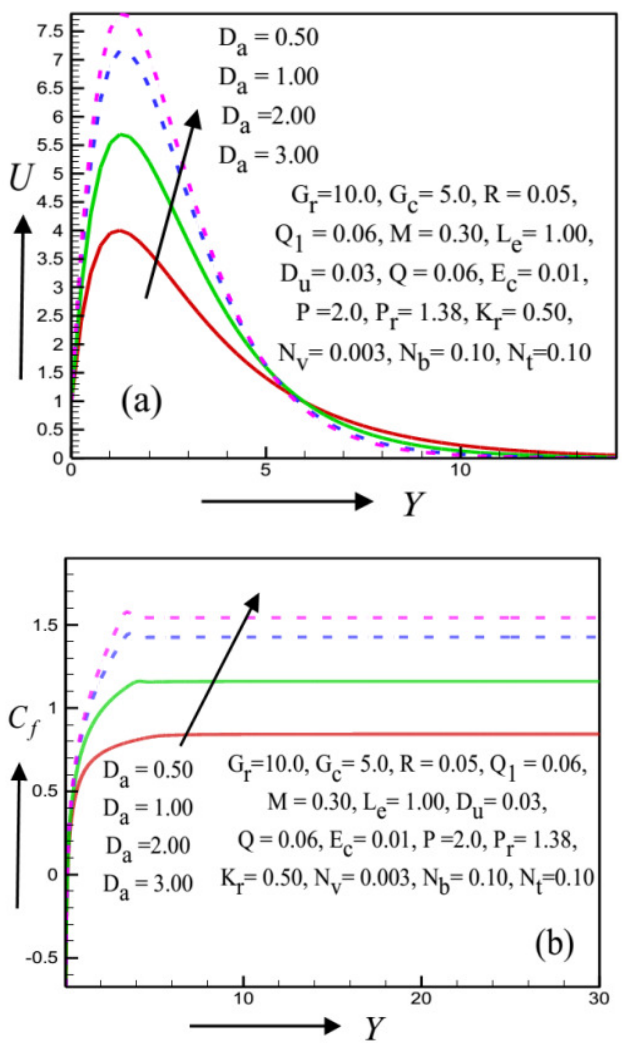

Fig. 3 Illustration of (a) velocity profiles for different values of $D_{a}$ and (b) skin friction for different values of $D_{a}$.

In Fig. 4(a) and Fig. 4(b) illustrate the variations of concentration and temperature profiles for various values of Brownian parameter $\left(N_{b}\right)$.It is found that the increase in the value of $N_{b} \geq 0.10$, the concentration boundary layer reduces and the thermal boundary layer increases. The numerical value obtained from the EFDM simulation for Brownian parameter $\left(N_{b}\right)$ varies from 0.10 to 0.60 . The curve to curve increment at $\mathrm{Y}=2.00$ are $50.22 \%, 13.81 \%$ and $4.82 \%$ increase for concentric boundary layer. On the other hand, thermal layer fluctuates $120.505 \%, 87.965 \%$ and $49.74 \%$ increase for Brownian parameter $\left(N_{b}\right)$ varies from 0.10 to 0.60 .
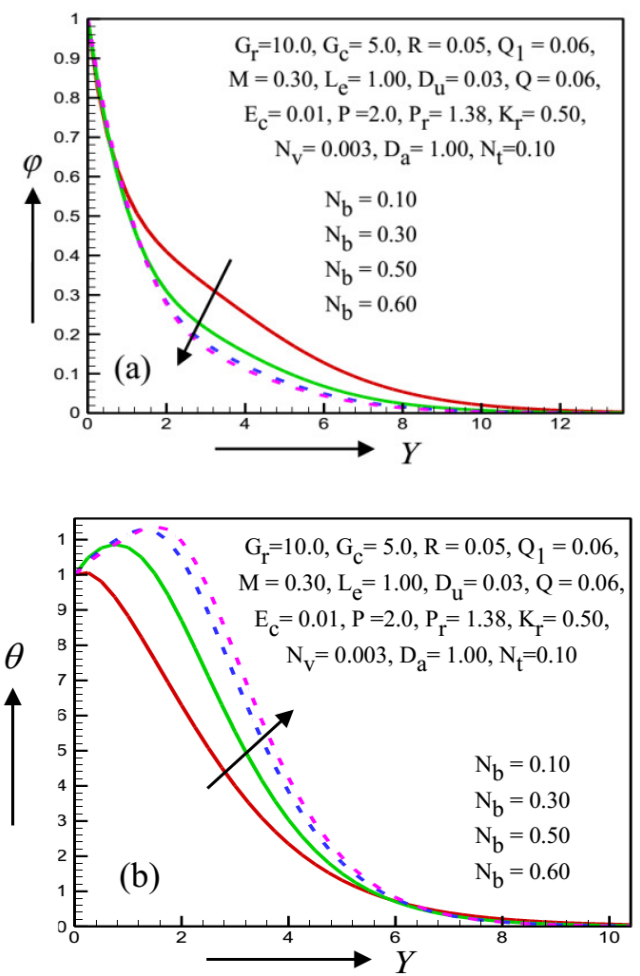

Fig. 4 Illustration of (a) concentration profiles for different values of $N_{b}$ and (b) temperature for different values of $N_{b}$.

In Fig. 5(a) and Fig. 5(b) illustrate the variations temperature and concentration profiles. With the increase of thermophoresis parameter $N_{t} \geq 0.10$, the thermal boundary layer profiles decrease. On the other hand, concentration boundary layer profiles increase rapidly. The first two curve fluctuation for the thermophoresis parameter $\left(N_{t}\right)$ variation is more than $100 \%$.
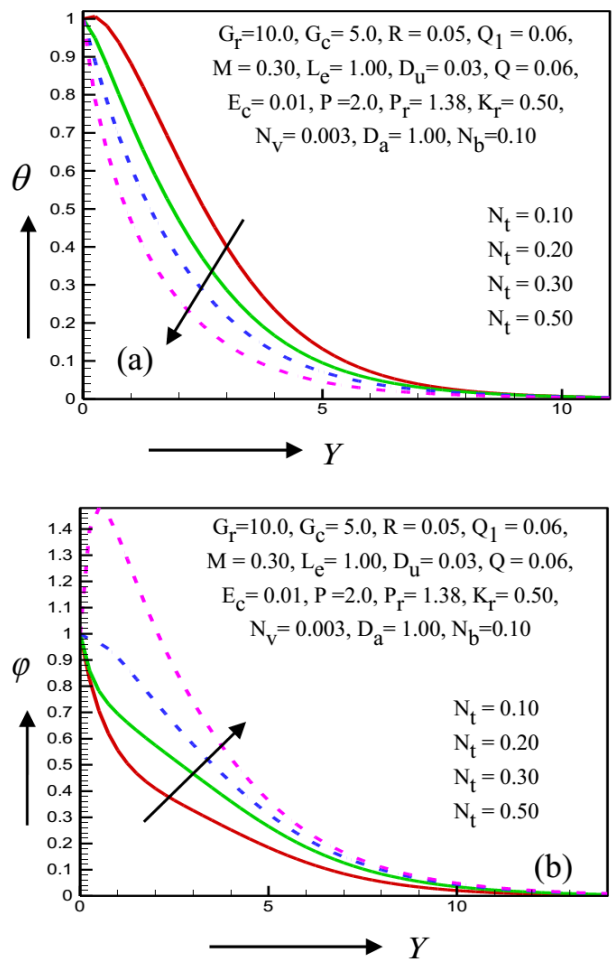

Fig. 5 Illustration of (a) temperature profiles for different $N_{t}$ and (b) concentration profiles for different values of $N_{t}$. 
Table 1 provides the numerical values of the local Nusselt number (Eq. (11)) obtained from the EFDM simulation for different thermophoresis parameter $\left(N_{t}\right)$ by changing $N_{b}$. The local Nusselt number is observed to be increase with increasing values of $N_{t}$ and $N_{b}$.

Table 1. Computations are showing the increase of Nusselt number $\left(N_{u}\right)$ for the increase of $N_{t}$ for $P_{r}=1.38, L_{e}=1.00$ and $\tau=1.20$.

\begin{tabular}{cccccc}
\hline$N_{t}$ & $\begin{array}{c}N_{u} \\
\left(N_{b}=\right.\end{array}$ & $\begin{array}{c}N_{u} \\
\left(N_{b}=\right.\end{array}$ & $\begin{array}{c}N_{u} \\
\left(N_{b}=\right.\end{array}$ & $\begin{array}{c}N_{u} \\
\left(N_{b}=\right.\end{array}$ & $\begin{array}{c}N_{u} \\
\left(N_{b}=\right.\end{array}$ \\
& $0.10)$ & $0.20)$ & $0.30)$ & $0.40)$ & $0.50)$ \\
\hline 0.10 & 0.07855 & 0.00310 & -0.02716 & -0.02451 & -0.02100 \\
0.20 & 0.14424 & 0.05009 & -0.01396 & -0.03049 & -0.01976 \\
0.30 & 0.21444 & 0.11994 & 0.03272 & -0.01688 & -0.01449 \\
0.40 & 0.28348 & 0.19285 & 0.09963 & 0.01639 & -0.01070 \\
0.50 & 0.33313 & 0.25967 & 0.17173 & 0.08377 & 0.00461 \\
\hline
\end{tabular}

Table 2 provides the numerical values of local Sherwood number (Eq. (12)) obtained from the EFDM simulation for different thermophoresis parameter $\left(N_{t}\right)$ by changing $N_{b}$. The local Sherwood number $\left(S_{h}\right)$ is decreased with the increment of $N_{t}$ and $N_{b}$.

Table 2. Computations are showing the decreased of Sherwood number $\left(S_{h}\right)$ for the increase of $N_{t}$ for $P_{r}=1.38, L_{e}=1.00$ and $\tau=1.20$.

\begin{tabular}{cccccc}
\hline$N_{t}$ & $\begin{array}{c}S_{h} \\
\left(N_{b}=\right.\end{array}$ & $\begin{array}{c}S_{h} \\
\left(N_{b}=\right.\end{array}$ & $\begin{array}{c}S_{h} \\
\left(N_{b}=\right.\end{array}$ & $\begin{array}{c}S_{h} \\
\left(N_{b}=\right.\end{array}$ & $\begin{array}{c}S_{h} \\
\left(N_{b}=\right.\end{array}$ \\
& $0.10)$ & $0.20)$ & $0.30)$ & $0.40)$ & $0.50)$ \\
\hline 0.10 & 0.17240 & 0.20418 & 0.29791 & 0.29378 & 0.28957 \\
0.20 & 0.06532 & 0.19508 & 0.22023 & 0.21174 & 0.24131 \\
0.30 & -0.15078 & 0.12756 & 0.21808 & 0.20774 & 0.22378 \\
0.40 & -0.46245 & -0.00146 & 0.16036 & 0.20217 & 0.21508 \\
0.50 & -0.81565 & -0.17724 & 0.06218 & 0.18273 & 0.20686 \\
\hline
\end{tabular}
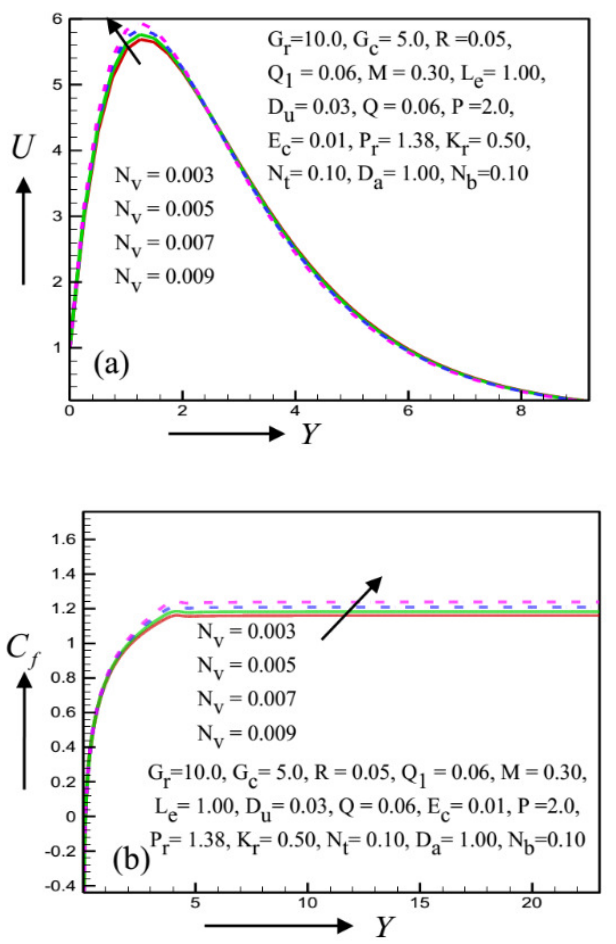

Fig. 6 Illustration of (a) velocity profiles for different values of $N_{v}$ and (b) skin friction profiles for different values of $N_{v}$.
In Fig. 6(a) and Fig. 6(b) illustrate the variations velocity and skin friction profiles with the effect of Maxwell parameter $\left(N_{v}\right)$. It can be seen that, $N_{v}$ has a significant impact on velocity boundary layer change. With the increase of $N_{v}$, the velocity and skin friction profiles increase rapidly. It is about more than $3000 \%$ for velocity and $1000 \%$ for skin friction profiles for the curve to curve fluctuation. This behavior occurred because of the Skin friction or friction loss is the loss of pressure that happens in pipe or duct flow for the effect of the fluid's viscosity near the surface of the pipe or duct. In the skin friction, the profiles pick up below to $X=1.2$ point and then remain unchanged.
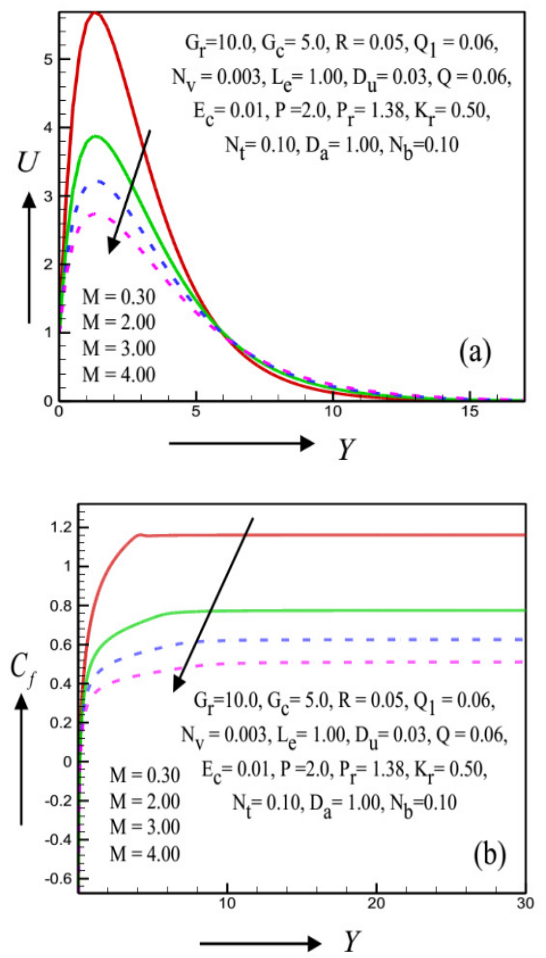

Fig. 7 Illustration of (a) velocity profiles for different values of $M$ and (b) skin friction profiles for different values of $M$.

The interaction of electrically conducting fluids with magnetic fields, through electromagnetic forces called Lorentz forces. Strong magnetic parameter $(M \geq 0.30)$ creates drag force known as Lorentz force and the force impact the fluid velocity to decrease.

In Fig. 7(a) and Fig. 7(b) exhibits the velocity and skin friction profiles for different $\mathrm{M}$. For the strong magnetic parameter, the velocity and skin friction profiles decrease because of the drag force effect. An immense change of $\mathrm{M}$ creates vast fluctuation of curve. For changing magnetic parameter $54.48 \%, 38.223 \%$ and $29.72 \%$ decrease for curve to curve at $\mathrm{Y}=3.00$. Also for the skin friction, the curves fluctuate occurs $22.85 \%, 15.156 \%$ and $11.866 \%$ decrease at $\tau=10.00$.

In Fig. 8(a) and Fig. 8(b), the variations of concentration and Sherwood number profiles are shown for different values of $K_{r}$. For the increase of destructive chemical reaction $\left(K_{r} \geq 0.50\right)$ parameter, the concentration boundary layer profiles decrease, and Sherwood number profiles increase. Increase of $K_{r}$ significantly alerts the concentration boundary layer thickness but does not alerts momentum boundary layer.

Fluid has two different transport phenomena, one is transport due to convection for the macroscopic fluid velocity and other is transport due to diffusion. In fluids both transport phenomena occur simultaneously. Viscosity is the main element for the fluid transport 
phenomenon. Lewis number $\left(L_{e}\right)$ is the ratio of the thermal diffusivity to mass diffusivity.
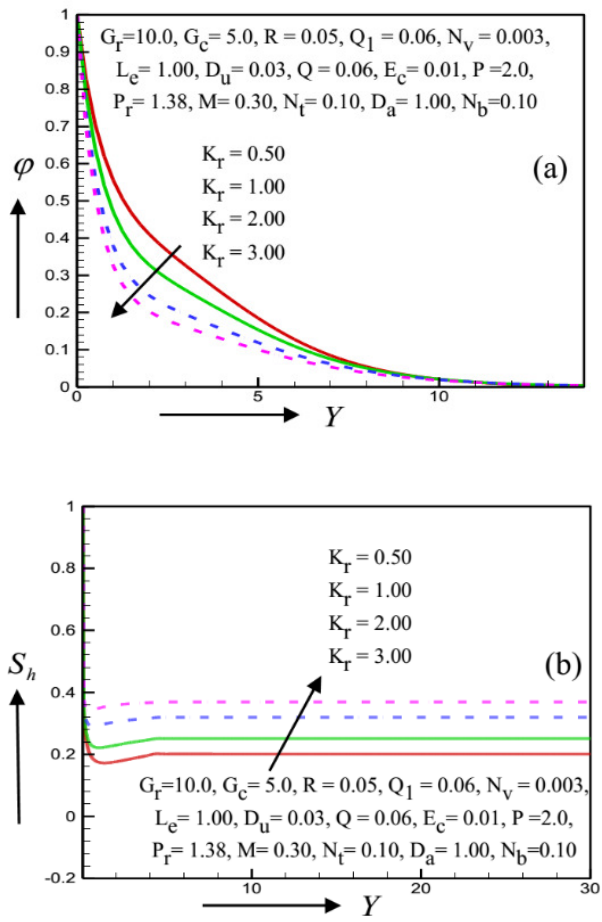

Fig. 8 Illustration of (a) concentration profiles for different $K_{r}$ and (b) Sherwood number profiles for different $K_{r}$.
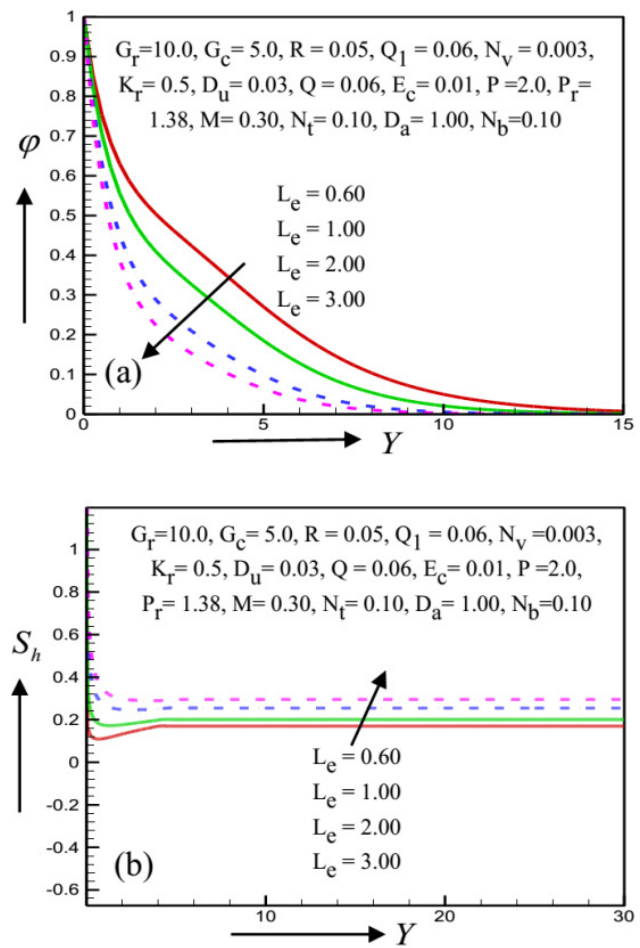

Fig. 9 Illustration of (a) concentration profiles for different $L_{e}$ and (b) Sherwood number profiles for different $L_{e}$.

Fig. 9(a) and Fig. 9(b) illustrates the variations of concentration and local Sherwood number profiles for different values of Lewis number, $L_{e}$. For the increase of $L_{e}$, concentration boundary layer profiles observed to be decrease rapidly whilst the local Sherwood number profiles increase. The numerical value obtained from the EFDM simulation for Lewis number in the curve to curve fluctuations decrease with $24.34 \%, 11.704 \%$ and $5.654 \%$ at $\mathrm{Y}=3.00$.
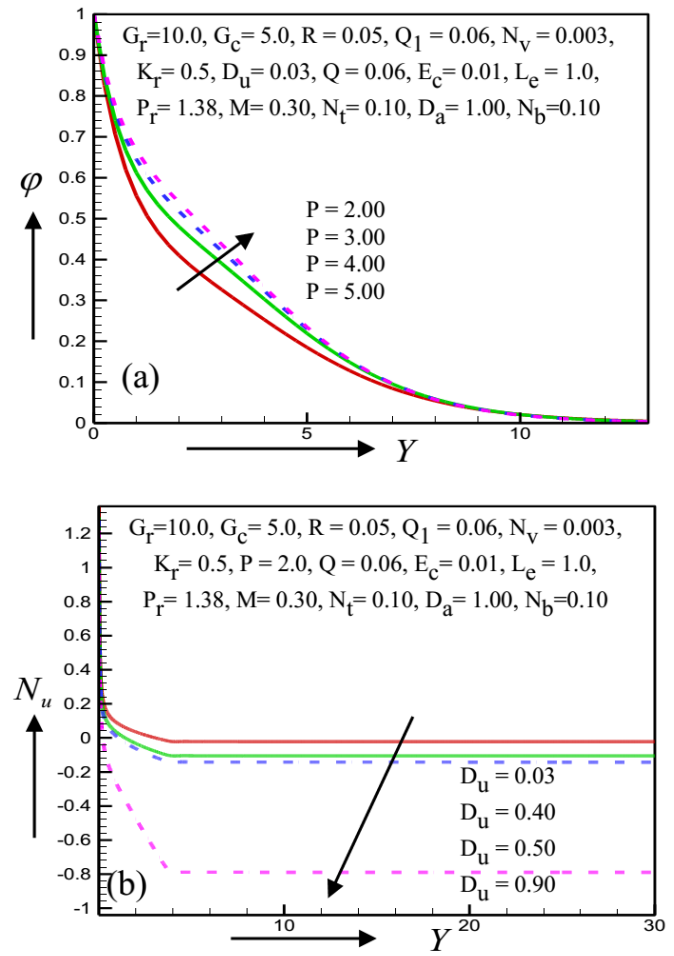

Fig. 10 Illustration of (a) concentration profiles for different values of $\mathrm{P}$ and (b) Nusselt number profiles for different $D_{u}$.

In Fig. 10(a) and Fig. 10(b) provides the variations of concentration and Nusselt number profiles for different values of $\mathrm{P}$ and $D_{u}$ respectively. Order of the destructive chemical reaction $(\mathrm{P})$ is observed to be influence the concentration profiles as it is increase due to increase in P. On the other hand, Mass diffusion gradient has a strong impact on heat transport phenomena. With the increase of $D_{u}$, Nusselt number profiles is found to be decrease. At $\mathrm{Y}=3.00$, the concentric boundary layer fluctuations curve to curve are $6.406 \%, 3.069 \%$ and $1.614 \%$ increase for order of the destructive chemical reaction. On the other hand, Nusselt number $\left(N_{u}\right)$ decrease around $3.09 \%, 36.63 \%$ and $163.71 \%$ for the increase of mass diffusion parameter. Fig. 11(a) - Fig. 12(a) displays the variations of velocity, temperature and skin friction profiles for different values of thermal radiation ( $R$ ) parameter. With the increase of $\mathrm{R}$, the velocity, temperature and skin friction profiles are also increase. Thermal radiation (electromagnetic radiation) could be attributed due to thermal excitation. The temperature could be affected in presence of thermal radiation at moderate temperatures which is significant. Thermal radiation for a medium which contains it inevitably has pressure and density gradients and the treatment requires the use of hydrodynamics. For the electromagnetic radiation by the thermal excitation increase the fluid velocity and it is maximum to near the plate. For the increment of $\mathrm{R}$, the velocity curves fluctuate $279.94 \%$, $243.34 \%$ and $209.43 \%$ and increase at a point $\mathrm{Y}=3.00$. On the other hand, thermal boundary layer fluctuates $51.28 \%$, $44.65 \%$ and $37.82 \%$.

Fig. 12(b) - Fig. 13(b) represent the effect of Prandtl number $\left(P_{r}\right)$ on temperature, local Nusselt and Sherwood number distributions. The parameter, $P_{r}$ is the ratio of kinematics viscosity to the thermal diffusivity which is physically very with temperature for example, water $P_{r}=7.0\left(\right.$ At $20{ }^{\circ} \mathrm{C}$ ), Ammonia gases $P_{r}=1.38$ falls more quickly compared to air $P_{r}=0.71$. In addition, $P_{r}<<1$ explains the thermal 
diffusivity dominates. For the large values of Prandtl number, i.e., $P_{r} \gg$ 1 , the momentum diffusivity dominates this behavior. With the increase of Prandtl number, temperature and Nusselt number $\left(N_{u}\right)$ profiles decrease whereas Sherwood number shows reverse properties.
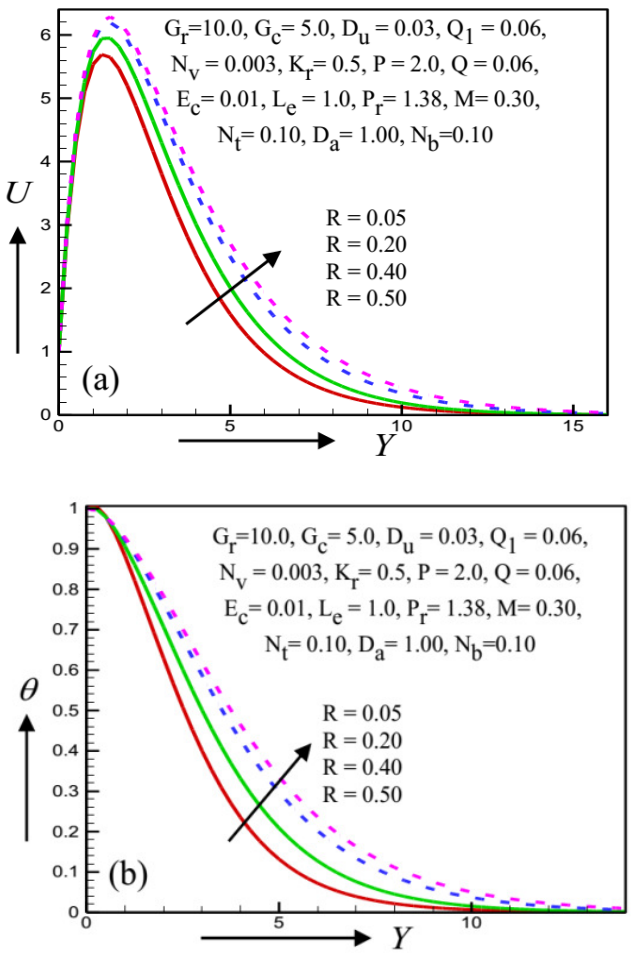

Fig. 11 Illustration of (a) velocity profiles for different values of $R$ and (b). temperature profiles for different values of $R$.
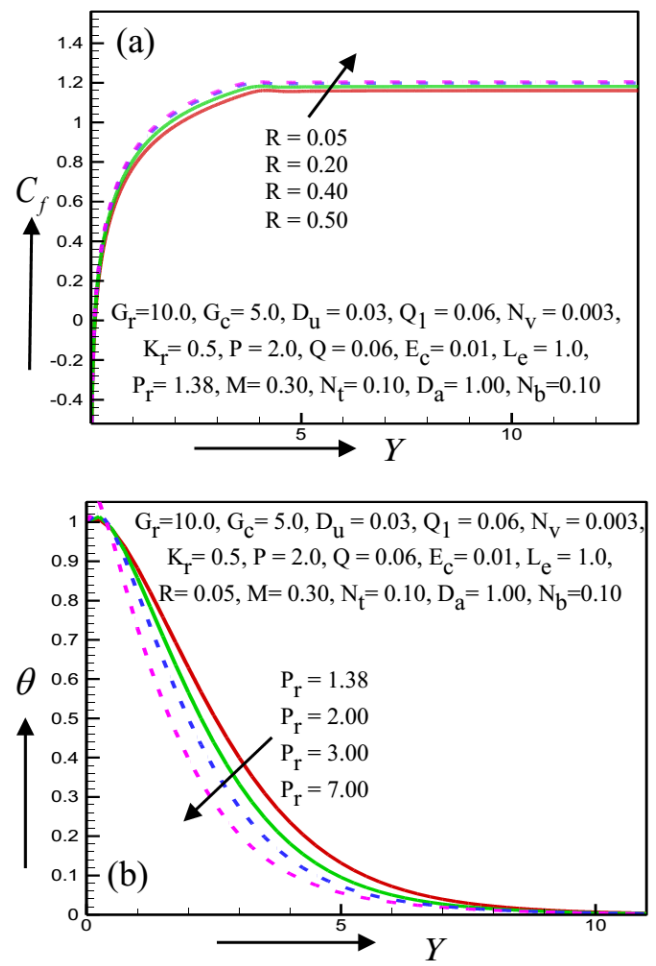

Fig. 12 Illustration of (a) skin friction profiles for different values of $R$ and (b) temperature profiles for different values of $P_{r}$.
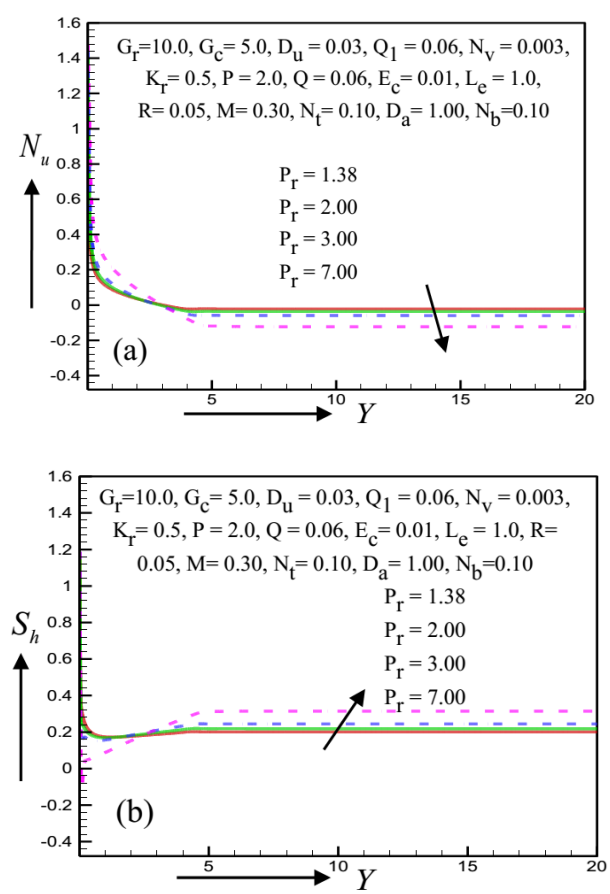

Fig. 13 Illustration of (a) Nusselt number profiles for different $P_{r}$ and (b) Sherwood number profiles for different $P_{r}$.
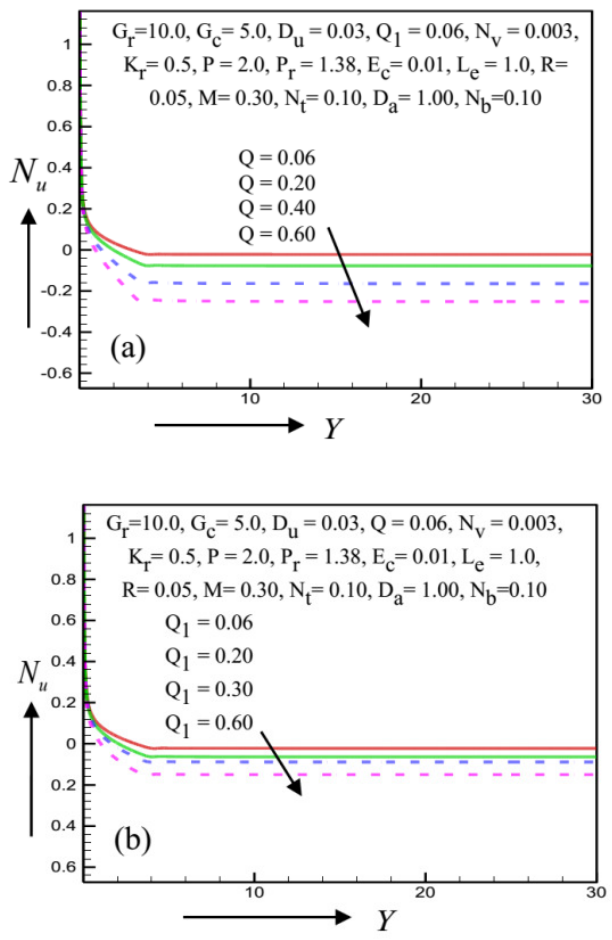

Fig. 14 Illustration of (a) Nusselt number profiles for different $Q$ and (b) Nusselt number profiles for different $\mathrm{Q}_{1}$.

Fig. 14(a) - Fig. 14(b) represent the effect of heat generation (Q) and radiation absorption $\left(Q_{1}\right)$ on Nusselt number profiles. With the increase of heat source and radiation absorption, Nusselt number profiles are observed to be decrease. With the numerical value obtained from the EFDM simulation it was observed that at $\tau=10.00$ dimensionless time, Nusselt number curves decrease at the rate of $110.8 \%, 43.14 \%$ and $43.30 \%$ for increasing $\mathrm{Q}$ whereas it decreases at the rate of $29.38 \%$, $25.12 \%$ and $20.52 \%$ for $Q_{1}$. 

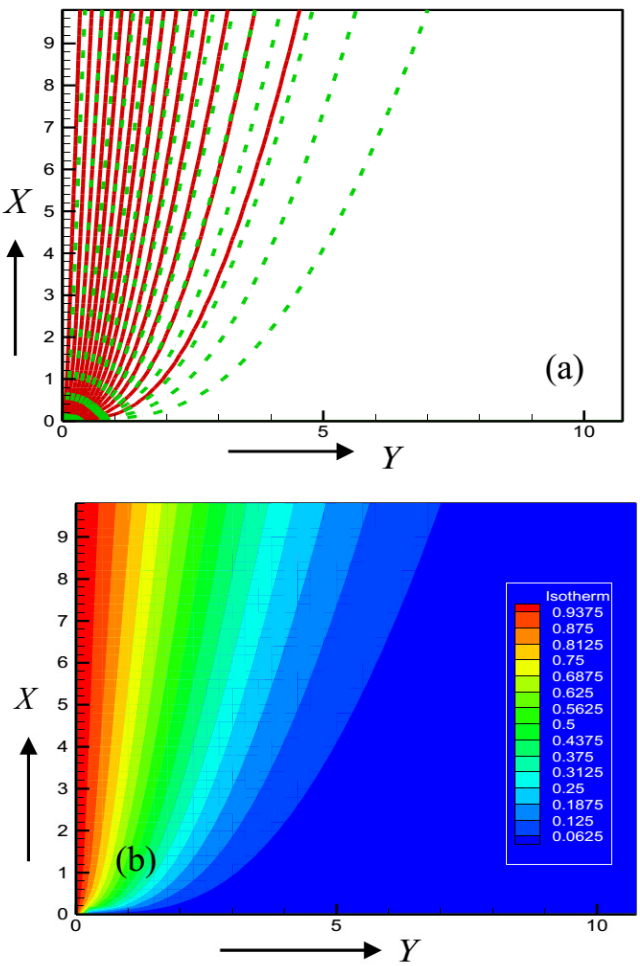

Fig. 15 Illustration of (a) Isotherms for $\mathrm{R}=0.05$ (red solid line) and $\mathrm{R}$ $=0.50$ (green dashed dot line) and (b) Isotherms flood view.

The non-dimensional equation after different transformations has been solved in the present numerical study. Therefore, for this reason, $\mathrm{X}$ and $\mathrm{Y}$ axis are dimensionless which indicates the mash point different from the numerical point of view. In addition, with the stream and isotherms (line view) curves, the difference of boundary layer for different parameters can be defined. The development of streamlines and isotherms are presented in Figs. 15-18. It can be observed that; thermal boundary layer and momentum boundary layer increases due to the increase of thermal radiation $(\mathrm{R})$. The legend values of stream and isotherms (flood view) indicate the contours levels. Furthermore, streamlines profiles can be used to improved visualization of fluid fields. It represents the velocity direction of fluids correspondingly. The streamlines can be obtained by drawing lines tangent to the flags. They are a rather mathematical object. They can be visualized by implanting little flags inside the fluid and observing their orientation. The boundary layer system of change can be shown by an isotherm, where the temperature remains constant $(\Delta T=0)$. An isotherm at $0^{0} C$ (the freezing point of water) is called the freezing level.

This phenomenon occurs when a system is in contact with an outside thermal layer (heat bath), and the change happens gradually enough to allow the system to continually adjust to the temperature of the boundary layer through heat exchange. Fig. 15 (a) displays the thermal direction of fluid (thermal boundary layer thickness) with the effect of presence of two different thermal radiation parameters. Where the red line indicates $\mathrm{R}=0.05$ and green line is for $\mathrm{R}=0.50$. Fig. 15 (b) shows the contour flood view for thermal radiation difference. The contours levels are also provided in the legend of Fig. 15 (b).

Fig. 16 (a) exhibits the isotherms plot of the momentum boundary layer thickness with the effect of presence of thermal radiation parameter where the red line is for $\mathrm{R}=0.05$ and green line is for $\mathrm{R}=$ 0.50 . Fig. 16 (b) shows the contour flood view for thermal radiation difference. The legend values of Fig. 16 (b) are given in the contours levels. The thermal and momentum boundary layer thickness is observed to be increases due to increase in $\mathrm{R}$.
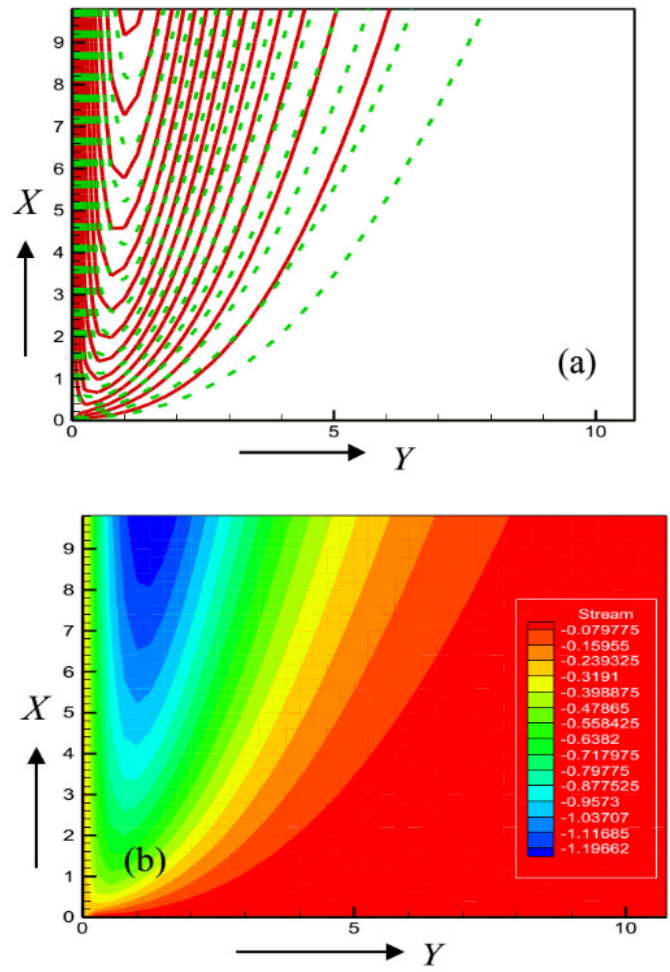

Fig. 16 Illustration of (a) Streamlines for $\mathrm{R}=0.05$ (red solid line) and $\mathrm{R}=0.50$ (green dashed dot line) and (b) Streamlines flood view.
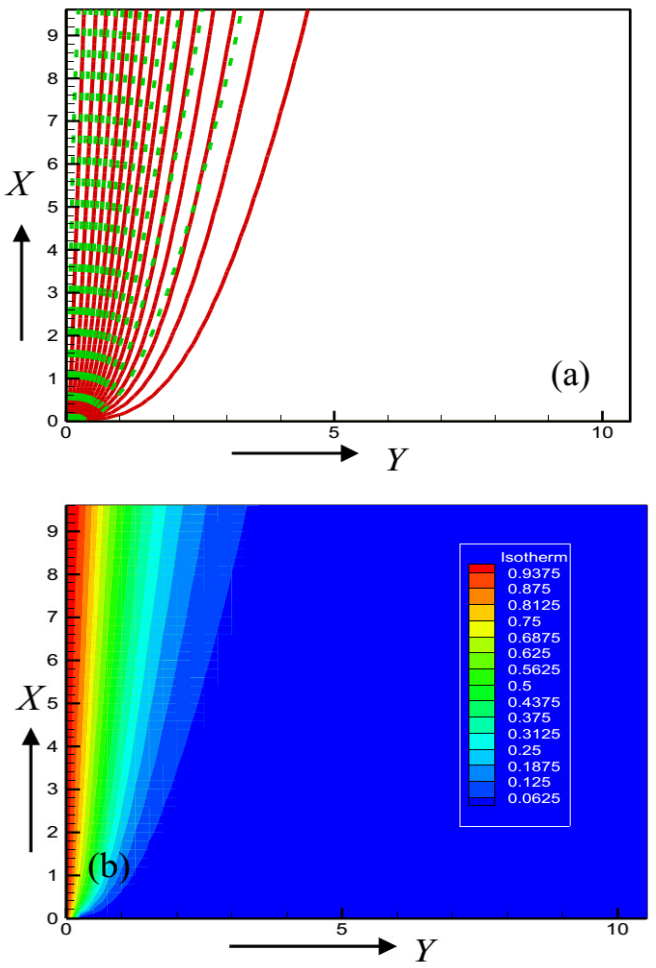

Fig. 17 Illustration of (a) Isotherms for $P_{r}=1.38$ (red solid line) and $P_{r}$ $=7.00$ (green dashed dot line) and (b) Isotherms flood view.

Fig. 17 (a) shows the thermal direction of fluid (thermal boundary layer thickness) with different Prandtl number $\left(P_{r}\right)$. The red line indicates $P_{r}=1.38$ whereas green line is for $P_{r}=7.00$. Fig. 17 (b) shows the contour flood view of Fig. 17 (a). The legend values of Figs. 17 (b) are the contours levels. 

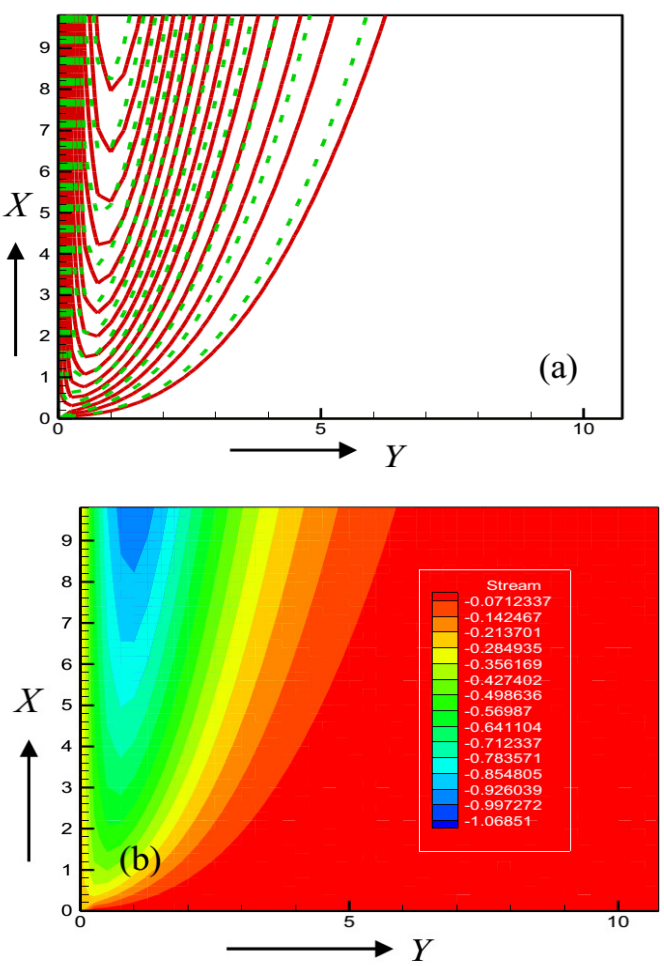

Fig. 18 Illustration of (a) Streamlines for $P_{r}=1.38$ (red solid line) and $P_{r}=7.00$ (green dashed dot line) and (b) Streamlines flood view.

Fig. 18 (a) shows the velocity direction of fluid (momentum boundary layer thickness) with the Prandtl number $\left(P_{r}\right)$ difference. In the graph, red line is for $P_{r}=1.38$ and green line is for $P_{r}=7.00$. Fig. 18 (b) shows the contour flood view for Prandtl number $\left(P_{r}\right)$ difference. The legend values of Fig. 18 (b) are also given in the contours levels. For the change of Prandtl number $\left(P_{r}\right)$ the thermal and momentum boundary layer thickness decrease.

\section{CONCLUDING REMARKS}

The Numerical solutions for Maxwell fluid with nano-particle towards a moving semi-infinite porous plate with thermal radiation, heat source, chemical reaction, mass diffusion is analyzed. The results are presented graphically with various parameters. Form the graphical representation, we have the following observations:

- Velocity profiles increase for increasing Darcy number, Maxwell parameter and thermal radiation individually however it decreases due to increase in magnetic parameter and Prandtl number.

- Temperature profiles found increase for individual rising of Brownian parameter, thermal radiation whilst it decreased due to increase in thermophoresis parameter and Prandtl number.

- Concentration profiles increase with the increase of individual Brownian parameter, thermophoresis parameter and chemical reaction order whereas profiles decrease with the rise of chemical reaction, Lewis number separately.

- Skin friction profiles increase for increasing of Darcy number, Maxwell parameter and thermal radiation respectively whilst it decreases with the increase magnetic parameter.

- Sherwood number profiles observe to be increased for the individual rising effects of chemical reaction, Lewis number and Prandtl number.

- Nusselt number profiles decrease for increasing mass diffusion, Prandtl number and heat and radiation absorption individually.

\section{NOMENCLATURE}

$B$

$C_{f}$

$C_{p}$

$D_{a}$

$D_{B}$

$D_{u}$

$E_{c}$

$G_{r}$

$G_{c}$

$K^{\prime}$

$K_{r}$

$k_{e}$

$L_{e}$

$N_{b}$

$N_{t}$

$N_{u}$

$N_{v}$

$P_{r}$

$q_{r}$

$Q_{1}{ }^{*}$

$Q$

$S_{h}$

$\mathrm{T}$

$\mathrm{T}_{\mathrm{W}}$

$U_{\circ}$

$u, v$

$x, y$

Greek symbols

$\rho$

$\mu$

$v$

$\beta$

$\beta^{*}$

$\kappa$

$\sigma_{s}$

Abbreviations

EFDM

ODE

PDE

SCA magnetic component, $\left(\mathrm{Wb} \mathrm{m}^{-2}\right)$

skin-friction, (-)

specific heat at constant pressure, $\left(\mathrm{J} \mathrm{kg}^{-1} \mathrm{~K}^{-1}\right)$

Darcy number, (-)

Brownian diffusion coefficient, (-)

Dufour number, (-)

Eckert number, (-)

Grashof number, (-)

modified Grashof number, (-)

permeability of the porous medium, (-)

chemical reaction parameter, (-)

mean absorption coefficient

Lewis number, (-)

Brownian parameter, (-)

thermophoresis parameter, (-)

local Nusselt number, (-)

Maxwell parameter

Prandtl number, (-)

unidirectional radiative heat flux, $\left(\mathrm{kg} \mathrm{m}^{-2}\right)$

radiation absorption, (-)

heat absorption quantity, (-)

Fluid temperature, $(\mathrm{K})$

Temperature at the plate surface, $(\mathrm{K})$

ambient temperature as y tends to infinity, $(\mathrm{K})$

uniform velocity

velocity components

Cartesian co-ordinates

density of the fluid, $\left(\mathrm{kg} \mathrm{m}^{-3}\right)$

dynamic viscosities

kinematic viscosity, $\left(\mathrm{m}^{2} \mathrm{~s}^{-1}\right)$

thermal expansion co-efficient

concentration expansion co-efficient

thermal conductivity, $\left(\mathrm{Wm}^{-1} \mathrm{~K}^{-1}\right)$

Stefan-Boltzmann constant, $5.6697 \times 10^{-8}$

$\left(\mathrm{W} / \mathrm{m}^{2} \mathrm{~K}^{4}\right)$

ordinary differential equation

partial differential equations

stability and convergence analysis
Sherwood number, (-)

explicit finite difference method

\section{REFERENCES}

Afify, A. A. and Elgazery, N. S., 2016, "Effect of a Chemical Reaction on Magnetohydrodynamic Boundary Layer Flow of a Maxwell Fluid over a Stretching Sheet with Nanoparticles," Particuology, 29, 154-161. http://dx.doi.org/10.1016/j.partic.2016.05.003

Arifuzzaman, S. M., Khan, M. S., Hossain, K. E., Islam, M. S., Akter, S. and Roy, R., 2017, "Chemically Reactive Viscoelastic Fluid Flow in Presence of Nano Particle through Porous Stretching Sheet," Frontiers in Heat and Mass Transfer, 9 (5), 1-12. http://dx.doi.org/10.5098/hmt.9.5

Baoku, I. G., Olajuwon, B. I. and Mustapha, A. O., 2013, "Heat and Mass Transfer on a MHD Third Grade Fluid with Partial Slip Flow Past an Infinite Vertical Insulated Porous Plate in a Porous Medium," International Journal of Heat and Fluid Flow, 40, 81-88. 


\section{http://dx.doi.org/10.1016/j.ijheatfluidflow.2013.01.016}

Bég, O. A., Khan, M. S., Karim, I., Alam, M. M. and Ferdows, M., 2014, "Explicit Numerical Study of Unsteady Hydromagnetic Mixed Convective Nanofluid Flow from an Exponentially Stretching Sheet in Porous Media," Applied Nanoscience, 4 (8), 943-957.

http://dx.doi.org/10.1007/s13204-013-0275-0

Benos, L. T., Kakarantzas, S. C., Sarris, I. E., Grecos, A. P. and Vlachos, N. S., 2014, "Analytical and Numerical Study of MHD Natural Convection in a Horizontal Shallow Cavity with Heat Generation," International Journal of Heat and Mass Transfer, 75, 1930.

http://dx.doi.org/10.1016/j.ijheatmasstransfer.2014.02.062

Biswas, P., Arifuzzaman, S. M., Rahman, M. M. and Khan, M. S., 2018, "Effects of Periodic Magnetic Field on 2D Transient Optically Dense Gray Nanofluid over a Vertical Plate: A Computational EFDM Study with SCA," Journal of Nanofluids, 7 (1), 82-91.

https://doi.org/10.1166/jon.2018.1434

Biswas, P., Arifuzzaman, S. M., Karim, I. and Khan, M.S., 2017, "Impacts of Magnetic Field and Radiation Absorption on Mixed Convective Jeffrey Nano Fluid Flow over a Vertical Stretching Sheet with Stability and Convergence Analysis," Journal of Nanofluids, 6 (6), 1082-1095.

https://doi.org/10.1166/jon.2017.1407

Chen, S., Zheng, L., Shen, B. and Chen, X., 2015, "Time-Space Dependent Fractional Boundary Layer Flow of Maxwell Fluid over an Unsteady Stretching Surface," Theoretical and Applied Mechanics Letters,5(6),262-266.

$\underline{\text { http://dx.doi.org/10.1016/j.taml.2015.11.005 }}$

Das, S., Jana, R. N. and Makinde, O. D., 2015, "Magnetohydrodynamic Mixed Convective Slip Flow over an Inclined Porous Plate with Viscous Dissipation and Joule Heating," Alexandria Engineering Journal, 54 (2), 251-261.

http://dx.doi.org/10.1016/j.aej.2015.03.003

Devi, S. P. A. and Kandasamy, R., 2002, "Effects of Chemical Reaction, Heat and Mass Transfer on Non-Linear MHD Laminar Boundary-Layer Flow over a Wedge with Suction or Injection," International Communications in Heat and Mass Transfer, 29 (5), 707716.

http://dx.doi.org/10.1016/S0735-1933(02)00389-5

Dogonchi, A. S. and Ganji, D. D., 2016, "Investigation of MHD Nanofluid Flow and Heat Transfer in a Stretching/Shrinking Convergent/Divergent Channel Considering Thermal Radiation," Journal of Molecular Liquids, 220, 592-603.

http://dx.doi.org/10.1016/j.molliq.2016.05.022

Durga Prasad, P., Kiran Kumar, R. V. M. S. S. and Varma, S. V. K., 2016, "Heat and Mass Transfer Analysis for the MHD Flow of Nanofluid with Radiation Absorption," Ain Shams Engineering Journal.

http://dx.doi.org/10.1016/j.asej.2016.04.016

Eid, M. R., 2016, “Chemical Reaction Effect on MHD Boundary-Layer Flow of Two-Phase Nanofluid Model over an Exponentially Stretching Sheet with a Heat Generation," Journal of Molecular Liquids, 220, 718725.

http://dx.doi.org/10.1016/j.molliq.2016.05.005
Elgazery, N. S., 2009, "The Effects of Chemical Reaction, Hall and Ion-Slip Currents on MHD Flow with Temperature Dependent Viscosity and Thermal Diffusivity," Communications in Nonlinear Science and Numerical Simulation, 14 (4), 1267-1283. http://dx.doi.org/10.1016/j.cnsns.2007.12.009

Ferdows, M., Khan, M. S., Bég, O. A. and Alam, M. M, 2013, "Numerical Study of Transient Magnetohydrodynamic Radiative Free Convection Nanofluid Flow from a Stretching Permeable Surface," Journal of Process Mechanical Engineering, 228 (3), 1-16. https://doi.org/10.1177/0954408913493406

Fetecau, C. and Zierep, J., 2003, “The Rayleigh-Stokes-Problem for a Maxwell Fluid," Z Angew Math Phys (ZAMP), 54, 1086-1093. http://doi.org/ 10.1007/s00033-003-1101-4

Ganga, B., Ansari, S. M. Y., Vishnu Ganesh, N. and Abdul Hakeem, A. K., 2015, "MHD Radiative Boundary Layer Flow of Nanofluid Past a Vertical Plate with Internal Heat Generation/Absorption, Viscous and Ohmic Dissipation Effects," Journal of the Nigerian Mathematical Society, 34(2),181-194.

http://dx.doi.org/10.1016/j.jnnms.2015.04.001

Hady, F. M., Mohamed, R. A. and Mahdy, A., 2006, "MHD Free Convection Flow Along a Vertical Wavy Surface with Heat Generation or Absorption Effect," International Communications in Heat and Mass Transfer,33(10),1253-1263.

http://dx.doi.org/10.1016/j.icheatmasstransfer.2006.06.007

Hayat, T., Abbas, Z. and Ali, N., 2008, "MHD Flow and Mass Transfer of a Upper-Convected Maxwell Fluid Past a Porous Shrinking Sheet with Chemical Reaction Species," Physics Letters A, 372 (26), 46984704.

http://dx.doi.org/10.1016/j.physleta.2008.05.006

Hayat, T., Abbas, Z. and Sajid, M., 2009, "MHD Stagnation-Point Flow of an Upper-Convected Maxwell Fluid over a Stretching Surface," Chaos, Solitons and Fractals, 39 (2), 840-848. http://dx.doi.org/10.1016/j.chaos.2007.01.067

Hayat, T., Fetecau, C. and Sajid, M., 2008, “On MHD Transient Flow of a Maxwell Fluid in a Porous Medium and Rotating Frame," Physics LettersA,372(10),1639-1644.

http://dx.doi.org/10.1016/j.physleta.2007.10.036

Hayat, T., Muhammad, T., Shehzad, S. A. and Alsaedi, A., 2017, “An Analytical Solution for Magnetohydrodynamic Oldroyd-B Nanofluid Flow Induced by a Stretching Sheet with Heat Generation/Absorption," International Journal of Thermal Sciences, 111, 274-288.

http://dx.doi.org/10.1016/j.ijthermalsci.2016.08.009

Hayat, T. and Qasim, M., 2010, "Influence of Thermal Radiation and Joule Heating on MHD Flow of a Maxwell Fluid in the Presence of Thermophoresis," International Journal of Heat and Mass Transfer, 53(21-22),4780-4788.

http://dx.doi.org/10.1016/j.ijheatmasstransfer.2010.06.014

Hayat, T. and Sajid, M., 2007, "Homotopy Analysis of MHD Boundary Layer Flow of an Upper-Convected Maxwell Fluid," International Journal of Engineering Science, 45 (2-8), 393-401. http://dx.doi.org/10.1016/j.ijengsci.2007.04.009

Hayat, T., Sajjad, R., Abbas, Z., Sajid, M. and Hendi, A. A., 2011, "Radiation Effects on MHD Flow of Maxwell Fluid in a Channel with Porous Medium," International Journal of Heat and Mass Transfer, 54 
(4),854-862.

http://dx.doi.org/10.1016/j.ijheatmasstransfer.2010.09.069

Hsiao, K.-L., 2017, “Combined Electrical MHD Heat Transfer Thermal Extrusion System Using Maxwell Fluid with Radiative and Viscous Dissipation Effects," Applied Thermal Engineering, 112, 1281-1288. http://dx.doi.org/10.1016/j.applthermaleng.2016.08.208

Ibáñez, G., López, A., Pantoja, J. and Moreira, J., 2016, "Entropy Generation Analysis of a Nanofluid Flow in MHD Porous Microchannel with Hydrodynamic Slip and Thermal Radiation," International Journal of Heat and Mass Transfer, 100, 89-97. http://dx.doi.org/10.1016/j.ijheatmasstransfer.2016.04.089

Ibrahim, F. S., Elaiw, A. M. and Bakr, A. A., 2008, "Effect of the Chemical Reaction and Radiation Absorption on the Unsteady MHD Free Convection Flow Past a Semi Infinite Vertical Permeable Moving Plate with Heat Source and Suction," Communications in Nonlinear Science and Numerical Simulation, 13 (6), 1056-1066.

http://dx.doi.org/10.1016/j.cnsns.2006.09.007

Islam, A., Islam, M. M., Rahman, M., Ali, L. E. and Khan, M. S., 2015, "Unsteady Heat Transfer of Viscous Incompressible Boundary Layer Fluid Flow Through a Porous Plate with Induced Magnetic Field," Journal of Applied Mathematics and Physics, 4, 294-306. http://dx.doi.org/10.4236/jamp.2016.42037

Kandasamy, R., Periasamy, K. and Sivagnana P. K. K., 2005, "Chemical Reaction, Heat and Mass Transfer on MHD Flow Over a Vertical Stretching Surface with Heat Source and Thermal Stratification Effects," International Journal of Heat and Mass Transfer, 48 (21-22), 4557-4561.

http://dx.doi.org/10.1016/j.ijheatmasstransfer.2005.05.006

Kandasamy, R., Vignesh, V., Kumar, A., Hasan, S. H. and Isa, N. M., 2016, "Thermal Radiation Energy Due to SWCNTS on MHD Nanofluid Flow in The Presence of Seawater/Water: Lie Group Transformation," Ain Shams Engineering Journal, In Press, Corrected Proof. http://dx.doi.org/10.1016/j.asej.2016.04.022

Khan, M. S., Wahiduzzaman, M., Karim, I., Islam, M. S. and Alam, M. M., 2014, "Heat Generation Effects on Unsteady Mixed Convection Flow from a Vertical Porous Plate with Induced Magnetic Field," Procedia Engineering, 90, 238-244.

http://dx.doi.org/10.1016/j.proeng.2014.11.843

Khan, N., Mahmood, T., Sajid, M., and Hashmi, M. S., 2016, "Heat and Mass Transfer on MHD Mixed Convection Axisymmetric Chemically Reactive Flow of Maxwell Fluid Driven by Exothermal and Isothermal Stretching Disks," International Journal of Heat and Mass Transfer, 92, 1090-1105.

http://dx.doi.org/10.1016/j.ijheatmasstransfer.2015.09.001

Mabood, F., Khan, W. A. and Ismail, A. I. M., 2015, "MHD Stagnation Point Flow and Heat Transfer Impinging on Stretching Sheet with Chemical Reaction and Transpiration," Chemical Engineering Journal, 273,430-437.

http://dx.doi.org/10.1016/j.cej.2015.03.037

Makinde, O. D. and Animasaun, I. L., 2016, "Bioconvection in MHD Nanofluid Flow with Nonlinear Thermal Radiation and Quartic Autocatalysis Chemical Reaction Past an Upper Surface of a Paraboloid of Revolution," International Journal of Thermal Sciences, 109, 159171.

http://dx.doi.org/10.1016/j.ijthermalsci.2016.06.003
Mohamed, R. A. and Abo-Dahab, S. M., 2009, "Influence of Chemical Reaction and Thermal Radiation on the Heat and Mass Transfer in MHD Micropolar Flow over a Vertical Moving Porous Plate in a Porous Medium with Heat Generation," International Journal of Thermal Sciences, $\mathbf{4 8}(9), 1800-1813$.

http://dx.doi.org/10.1016/j.ijthermalsci.2009.01.019

Mosayebidorcheh, S., Sheikholeslami, M., Hatami, M. and Ganji, D. D., 2016, "Analysis of Turbulent MHD Couette Nanofluid Flow and Heat Transfer Using Hybrid DTM-FDM,” Particuology, 26, 95-101. http://dx.doi.org/10.1016/j.partic.2016.01.002

Murthy, M. V. R., Raju, R. S. and Rao, J. A., 2015, "Heat and Mass Transfer Effects on MHD Natural Convective Flow Past an Infinite Vertical Porous Plate with Thermal Radiation and Hall Current," Procedia Engineering, 127, 1330-1337. http://dx.doi.org/10.1016/j.proeng.2015.11.491

Muthuraj, R., Nirmala, K. and Srinivas, S., 2016, "Influences of Chemical Reaction and Wall Properties on MHD Peristaltic Transport of a Dusty Fluid with Heat and Mass Transfer," Alexandria Engineering Journal,55(1),597-611. http://dx.doi.org/10.1016/j.aej.2016.01.013

Nandy, S. K. and Mahapatra, T. R., 2013, "Effects of Slip and Heat Generation/Absorption on MHD Stagnation Flow of Nanofluid Past a Stretching/Shrinking Surface with Convective Boundary Conditions," International Journal of Heat and Mass Transfer, 64, 1091-1100. http://dx.doi.org/10.1016/j.ijheatmasstransfer.2013.05.040

Nayak, M. K., Dash, G. C. and Singh, L. P., 2016, "Heat and Mass Transfer Effects on MHD Viscoelastic Fluid over a Stretching Sheet Through Porous Medium in Presence of Chemical Reaction," Propulsion and Power Research, 5 (1), 70-80.

http://dx.doi.org/10.1016/j.jppr.2016.01.006

Pandey, A. K. and Kumar, M., 2016, "Effect of Viscous Dissipation and Suction/Injection on MHD Nanofluid Flow over a Wedge with Porous Medium and Slip," Alexandria Engineering Journal, 55 (4), 3115-3123. http://dx.doi.org/10.1016/j.aej.2016.08.018

Rahman, M. M., Khan, M. S. and Islam, M. R., 2014, "Numerical Investigation on MHD Fluid Flow in the Presence of Chemical Reaction with Soret and Dufour Effects over a Vertical Plate in a Rotating System," Journal of Applied Physical Science International, 4(2), 77-88.

Ramachandra Prasad, V., Vasu, B. and Bég, O. A., 2011, “ThermoDiffusion and Diffusion-Thermo Effects on MHD Free Convection Flow Past a Vertical Porous Plate Embedded in a Non-Darcian Porous Medium," Chemical Engineering Journal, 173 (2), 598-606. http://dx.doi.org/10.1016/j.cej.2011.08.009

Ramesh, G. K., Gireesha, B. J., Hayat, T. and Alsaedi, A., 2016, "Stagnation Point Flow of Maxwell Fluid Towards a Permeable Surface in the Presence of Nano Particles," Alexandria Engineering Journal, 55, 857-865.

http://doi.org/10.1016/j.aej.2016.02.007

Ravikumar, V., Raju, M. C. and Raju, G. S. S., 2014, "Combined Effects of Heat Absorption and MHD on Convective Rivlin-Ericksen Flow Past a Semi-Infinite Vertical Porous Plate with Variable Temperature and Suction," Ain Shams Engineering Journal, 5 (3), $867-$ 875. http://dx.doi.org/10.1016/j.asej.2013.12.014 
Reddy, J. V. R., Sugunamma, V., Sandeep, N. and Sulochana, C., 2016, Influence of Chemical Reaction, Radiation and Rotation on MHD Nanofluid Flow Past a Permeable Flat Plate in Porous Medium," Journal of the Nigerian Mathematical Society, 35 (1), 48-65. http://dx.doi.org/10.1016/j.jnnms.2015.08.004

Reddy, P. B. A., 2016, "Magnetohydrodynamic Flow of a Casson Fluid over an Exponentially Inclined Permeable Stretching Surface with Thermal Radiation and Chemical Reaction," Ain Shams Engineering Journal,7(2),593-602. http://dx.doi.org/10.1016/j.asej.2015.12.010

Reddy, P. S. and Chamkha, A. J., 2016, "Soret and Dufour Effects on MHD Convective Flow of $\mathrm{Al}_{2} \mathrm{O}_{3}-$ Water and Tion-Water Nanofluids Past a Stretching Sheet in Porous Media with Heat Generation/Absorption," Advanced Powder Technology, 27 (4), 1207 1218. http://dx.doi.org/10.1016/j.apt.2016.04.005

Reddy, P. S., Sreedevi, P. and Chamkha, A. J., 2017, "MHD Boundary Layer Flow, Heat and Mass Transfer Analysis over a Rotating Disk Through Porous Medium Saturated by $\mathrm{Cu}-$ Water and Ag-Water Nanofluid with Chemical Reaction," Powder Technology, 307, 46-55. http://dx.doi.org/10.1016/j.powtec.2016.11.017

Reddy, V. P., Kumar, R. V. M. S. S. K., Reddy, G. V., Prasad, P. D. and Varma, S. V. K., 2015, "Free Convection Heat and Mass Transfer Flow of Chemically Reactive and Radiation Absorption Fluid in an Aligned Magnetic Field," Procedia Engineering, 127, 575-582.

http://dx.doi.org/10.1016/j.proeng.2015.11.347

Rubio Hernández, V. and Zueco, J., 2013, "Network Numerical Analysis of Radiation Absorption and Chemical Effects on Unsteady MHD Free Convection Through a Porous Medium," International Journal of Heat and Mass Transfer, 64, 375-383. http://dx.doi.org/10.1016/j.ijheatmasstransfer.2013.03.074

Satya Narayana, P. V., 2015, "Effects of Variable Permeability and Radiation Absorption on Magnetohydrodynamic (MHD) Mixed Convective Flow in a Vertical Wavy Channel with Traveling Thermal Waves," Propulsion and Power Research, 4 (3), 150-160.

http://dx.doi.org/10.1016/j.jppr.2015.07.002

Satya Narayana, P. V., Venkateswarlu, B. and Venkataramana, S., 2013, "Effects of Hall Current and Radiation Absorption on MHD Micropolar Fluid in a Rotating System," Ain Shams Engineering Journal, 4(4),843-854.

http://dx.doi.org/10.1016/j.asej.2013.02.002

Shahmohamadi, H. and Rashidi, M. M., 2016, "VIM Solution of Squeezing MHD Nanofluid Flow in a Rotating Channel with Lower Stretching Porous Surface," Advanced Powder Technology, 27 (1), 171178.

http://dx.doi.org/10.1016/j.apt.2015.11.014

Sheikholeslami, M., Abelman, S. and Ganji, D. D., 2014, "Numerical Simulation of MHD Nanofluid Flow and Heat Transfer Considering Viscous Dissipation," International Journal of Heat and Mass Transfer, 79,212-222.

http://dx.doi.org/10.1016/j.ijheatmasstransfer.2014.08.004

Sheikholeslami, M., Rashidi, M. M., Al Saad, D. M., Firouzi, F., Rokni, H. B. and Domairry, G., 2016, "Steady Nanofluid Flow Between Parallel Plates Considering Thermophoresis and Brownian Effects," Journal of King Saud University - Science, 28 (4), 380-389. http://dx.doi.org/10.1016/j.jksus.2015.06.003
Sreedevi, G., Raghavendra Rao, R., Prasada Rao, D. R. V. and Chamkha, A. J., 2016, "Combined Influence of Radiation Absorption and Hall Current Effects on MHD Double-Diffusive Free Convective Flow Past a Stretching Sheet," Ain Shams Engineering Journal, 7 (1), 383-397.

http://dx.doi.org/10.1016/j.asej.2015.11.024

Srinivas, S., Vijayalakshmi, A., Subramanyam Reddy, A. and Ramamohan, T. R., 2016, "MHD Flow of a Nanofluid in an Expanding or Contracting Porous Pipe with Chemical Reaction and Heat Source/Sink," Propulsion and Power Research, 5 (2), 134-148.

\section{http://dx.doi.org/10.1016/j.jppr.2016.04.004}

Srinivasa, A. H. and Eswara, A. T., 2016, "Effect of Internal Heat Generation or Absorption on MHD Free Convection from an Isothermal Truncated Cone," Alexandria Engineering Journal, 55 (2), 1367-1373. http://dx.doi.org/10.1016/j.aej.2016.04.003

Thomas P., 2010, "Nanoparticles Emit EM Radiation to Enhance Thermal Conductivity and Boiling Heat Transfer," PRLog. https://www.prlog.org/10495522-nanoparticles-emit-em-radiation-toenhance-thermal-conductivity-and-boiling-heat-transfer.html

Umamaheswar, M., Raju, M. C., Varma, S. V. K. and Gireeshkumar, J., 2016, "Numerical Investigation of MHD Free Convection Flow of a Non-Newtonian Fluid Past an Impulsively Started Vertical Plate in the Presence of Thermal Diffusion and Radiation Absorption," Alexandria Engineering Journal, 55 (3), 2005-2014.

http://dx.doi.org/10.1016/j.aej.2016.07.014

Zhang, Y. and Zheng, L., 2012, "Analysis of MHD Thermosolutal Marangoni Convection with the Heat Generation and a First-Order Chemical Reaction," Chemical Engineering Science, 69 (1), 449-455. http://dx.doi.org/10.1016/j.ces.2011.10.069

\section{APPENDIX A}

To obtain the steady-state solutions of the computation, the calculation has been carried out up to non-dimensional time, $\tau=10$ to 50. The velocity, temperature and concentration profiles shows a minor change after non-dimensional time, $\tau=30$ (Fig. A1). Therefore, the solution for $\tau \geq 30$ is considered as steady-state solution.

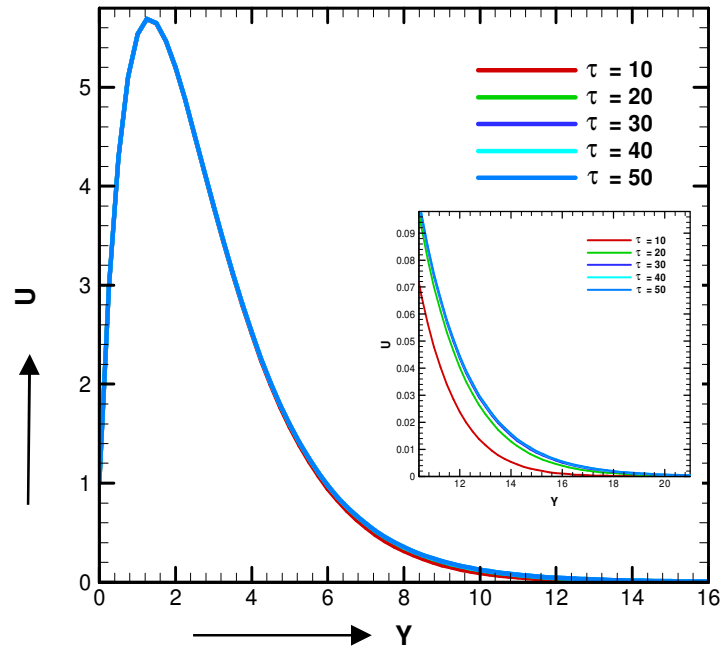

Fig. A1. Illustration of velocity profiles for different dimensionless time steps, $\tau=10$ to 50 , where, $\mathrm{G}_{\mathrm{r}}=10.0, \mathrm{G}_{\mathrm{c}}=5.0, \mathrm{D}_{\mathrm{u}}=0.03, \mathrm{Q}_{1}=$ $0.06, \mathrm{~N}_{\mathrm{v}}=0.003, \mathrm{~K}_{\mathrm{r}}=0.5, \mathrm{P}=2.0, \mathrm{P}_{\mathrm{r}}=1.38, \mathrm{E}_{\mathrm{c}}=0.01, \mathrm{~L}_{\mathrm{e}}=1.0, \mathrm{R}=$ $0.05, \mathrm{M}=0.30, \mathrm{~N}_{\mathrm{t}}=0.10, \mathrm{D}_{\mathrm{a}}=1.00, \mathrm{~N}_{\mathrm{b}}=0.10$. 